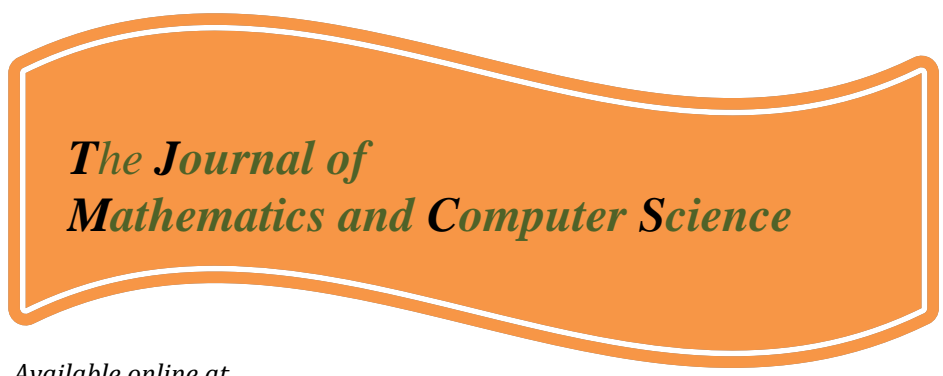

Available online at

http://www.TJMCS.com

The Journal of Mathematics and Computer Science Vol .1 No.4 (2010) 413-434

\title{
Integrated Fuzzy VIKOR and Fuzzy AHP Model for Supplier Selection in an Agile and Modular Virtual Enterprise; Application of FMCDM on Service Companies
}

\author{
Peyman mohammady ${ }^{1,}{ }^{*}$, Amin Amid ${ }^{2}$ \\ shahidbeheshti university,Pa.mohammady@yahoo.com \\ shahidbeheshti university, A_amid@sbu.ac.ir
}

Received: August 2010, Revised: November 2010

Online Publication: December 2010

\begin{abstract}
In this ever-changing world, organizations need to Outsource parts of their processes for having agile response to market's needs and varying demands. Because of temporal nature of virtual enterprises(VE's), the situation of outsourcing process in this kind of organizations is a vital situation. The main aim of this paper is to present a decision-making framework for specific area that is appropriated for complex states. The contribution of this paper is developing of fuzzy VIKOR method and combining it with fuzzy AHP. This extension suitable for decision-making situations whichbe face with mixture appraisement that simultaneously regarded to both "group utility" or majority and "individual regret" of the opponent. The Integrated and developed model is suitable for inconsistent conditions that we face to collection of criterias and sub criterias that should satisfy some of them collectively and simultaneously and in other attainment of some individual criterias is desirable. This framework then extended to a case study with varied criterias for outsourcing process.
\end{abstract}

Keywords:Fuzzy AHP, Fuzzy VIKOR, Multicriteria decision-making, Virtual Enterprises

1* Corresponding author: Master's student, Information Technology management, Faculty of management and accounting, Tehran, I.R. Iran

2- Assistant professor of faculty of management and accounting, Tehran, I.R. Iran 


\section{Introduction}

In among of organizations, VE's have higher complexity because of dynamic and temporal nature. The different definitions of VEhave been used in researches. Camarinha-Matosetal.(1997) introduce it as a temporal alliance that organizations based on it, share their competencies and resources [1].Gazendam(2001) define it as multi-actor systems that composed from human resources and virtual factors [2]. There are few differences between VE's and other form of organizations. Putniket al.(2005) summarize them to three fundamental features: dynamicsof network reconfiguration, virtuality and external entities[3]. VE's have shorter life cycle than others. As illustrated in Fig. 1, the life cycle of VEs is depended on business opportunities or need to adoption with changing environment.

In current hyper competition era, some philosophies and concepts are inevitable for VEs' as temporal alliance through it. Agility, modularity, and interoperation are of such concepts.For agility concept, different definitionshave been presented. Although common aspect of them is emphasize on flexibility and speed as primary properties of agility [4] and [5]. An important characteristic that has been considered in dominant researches is proactive and speedy response to change and [6][8].

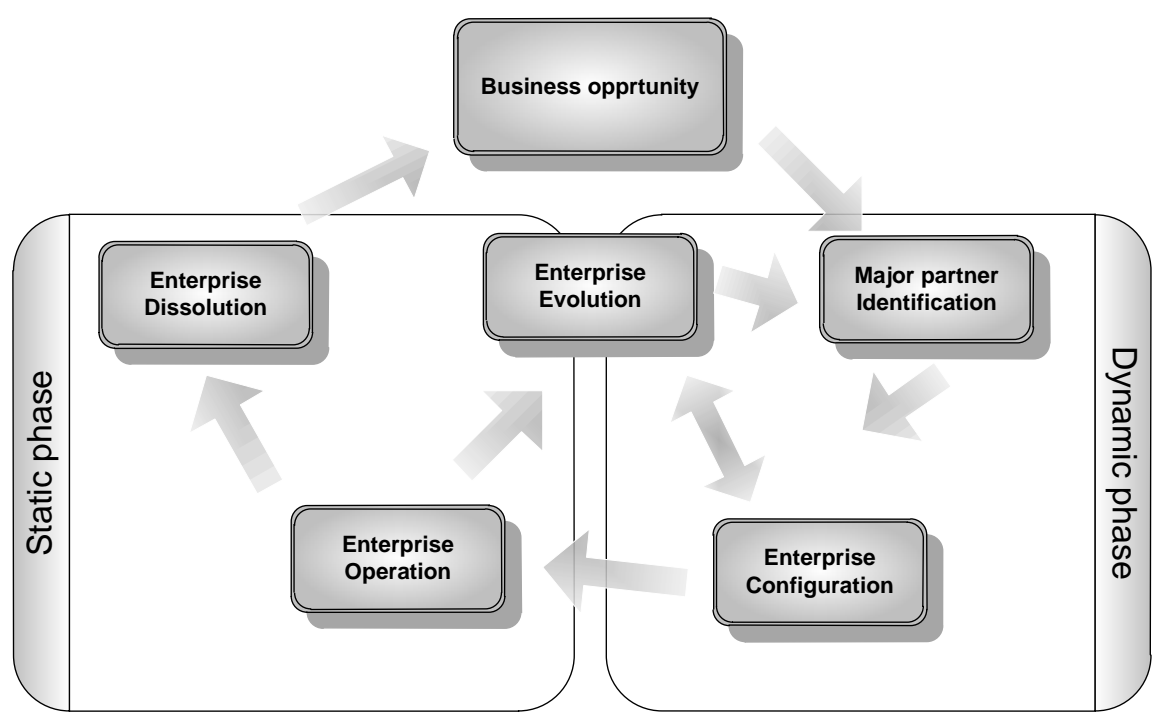

Figure 1. The life cycle of VE, Kim etal. (2006)[9]

At changing environment, the modularity concept is a critical philosophy for masscustomization production. This concept related to agile production As some researcher such Anderson(1997) showedwith higher levels of modularity, higher levels of flexibility and compatibility are attainable[10]. However this concept is less studied in the services sector. The vital role of modularity is related to postponement strategy and combination of both lean and agile philosophies. Note that modular products and services don't depend on modular processes only but modular supply chain had important impact on it. Lau et al. (2007) approved relation between supply chain integration processeswith modular products[11]. This finding is consistent with fine's (1998) research [12]. Corvello and Migliarese(2007) point out if processes haven't modularity characteristics, it is impossible to find suppliers with competencies for doing production phases [13]. Baldwin and Clarck (1997) describe modularity concept as complex product and process composed of series of sub-systems that each of them design independently and act with together as 
whole[14]. This definition is consistent withtowill's definition of agility referee to efforts aggregation for component's seamless of supply chain due to perceive themselves as whole [15]. This means joint and linkage of agility, interoperability and modularity concepts in VE's is establishment of seamless suppliers in supply chain of VE. Thus as cited in [16], selection's process of supplier(s) has a vital situation in outsourcing process. This importance may be because of supply chain and production strategies' affecting from this selection.

In outsourcing process of agile and modular virtual enterprise, we face with two kind of main criterias. First category including sub-criterias from "agility' criteria and next related to subcriterias from "modularity" criteria for supplier's selection. According to Qumer and Henderson's (2006) 4-DAT we can extract and define sub-criterias of agility level. 4-DAT propose framework to evaluate agility of software's development methods[17]. For virtual enterprise with IS/IT context, this framework is useful and can be used for construction of first level. With attention to this, outsourcing process have two aspects; first is extraction and determination of processes that should outsource and next is supplier's evaluation and selection for each or collection of these processes; we need to select some of commensurable criterias inevitably for context of virtual enterprise supply chain. From other hand such criterias should compatible with modularity's preservation goal. With analysis criterias based on cited aspects, the suitable sub-criterias are flexibility, speed, leanness, learning and responsiveness. However modularity has multidisciplinary but schilling (2003) categorized this concept and cite several properties of concept at different domain[18]. Among of These properties, some characteristicsare suitable for virtual enterprise with software development context. it including re-combinability, expandability, decomposability and module as homologue. These properties absolutely are compatible with VE's life cycle. We eliminate last property because of its concept is goal of outsourcing process in this context. As discussed above, the main goal of evaluation is seamless preservation due to guarantee all of supply chain components and so Virtual enterprise remains agile and modular.

\section{MCDM}

Multicriteria optimization (MCO) is considered as the process of determining the best feasible solution according to established criteria which represent different effects. However, these criteria usually conflict with each other and in Practical problems are often characterized by several noncommensurable or competing criteriaseverit may be there is no solution satisfying all criteria simultaneously. Multicriteria decision-making (MCDM) at new changing and turbulent environment should deal with Decision-making situations with uncertainty and non-crisp conditions. Thus several techniques and methods have been introduced and discussed for dealing with imprecise, uncertain, and complex decision-making problems; researchers have proposed different multicriteria decision-making (MCDM) approaches, such as the technique for order preference by similarity to ideal solution (TOPSIS). Further studies have extended MCDM in a fuzzy environment and proposed varied fuzzy multi-criteria decision-making (FMCDM) methods or other advanced techniques. These effective proposed techniques connected decision making with fuzzy set applications, to solve the problem for the optimal selectionsuch as the VlseKriterijumskaOptimizacija I KompromisnoResenje (VIKOR), fuzzy TOPSIS and fuzzy AHP.Each of these methods has its context and need to perceive along it. First wewill describe fuzzy VIKOR and fuzzy AHP summarily below. Next we develop fuzzy VIKOR for specific and multi-purpose situations and combine it with pairwise comparisons as a core of it. Detailed calculations, integrated and extended algorithm presented in appendix 1.

\subsection{Fuzzy VIKOR}


The VIKOR method was developed for multi-criteria optimization of complex systems. This method first time was developed by opricovic in 1998 to solve MCDM problems with conflicting and non-commensurable criteria [19]. This method focuses on ranking and selecting from a set of alternatives in the presence of conflicting criteria. It determines the compromise ranking-list, the compromise solution, and the weight stability intervals for preference stability of the compromise solution obtained with the initial (given) weights. It introduces an aggregating function representing the distance from the ideal solution. This ranking index is an aggregation of all criteria, the relative importance of the criteria, and a balance between total and individual satisfaction [20]. The main distinctive characteristic of fuzzy VIKOR is authority of decision-making based on group utility or individual regret of the opponent. According to VIKOR's algorithm we can select alternatives with considering two viewpoints. Upon on these states, it may be we want to select alternatives with higher scores based on all of criterias or ranked alternatives considering distinctive and higher distance from other alternatives in one or several criterias. It is suitable for problems and situations that we face with criterias including of several sub-criterias and we want to have appropriate decisions with flexibility of group utility or individual regret of the opponent.In real conditions, It's may be some criterias can be attainable with attaining of maximum of subcriterias and some criteria have specific construct which can be attainable by attaining each of subcriteria independently and in absence of other sub-criteria. Main advantage of fuzzy VIKOR rather than other is such distinction. This method applieda numerical weight to percentage for shift between two cited philosophy which refereed to environment and context of organization and its appraisement.Note that one of two states can be attained and in some context that we face with all of these states fuzzy VIKOR method don't appropriate for them. We developed this method for covering of such problems -Inconsistent situations that some criterias and their sub-criterias should simultaneously are attainable and some criteriascan be attained based on attaining of one or several their sub-criterias. The main contribution of this paper is relevant to this domain and then combination of it to fuzzy AHP methods.

\subsection{Fuzzy AHP}

The Analytic Hierarchy Process (AHP) introduced by saaty (1980), directs how to determine the priority of a set of alternatives and the relative importance of attributes in a Multicriteria decision-making problems [21]. Through AHP, the importance of several attributes is obtained from a process of paired comparison, in which the relevance of the attributes' class or drivers' categories of intangible assets are matched two-on-two in a hierarchic structure. However as cited by Yang and Chen's research(2004), the pure AHP model has some shortcomings. They pointed out that the AHP method is mainly used in nearly crisp-information decision applications; the AHP method creates and deals with a very unbalanced scale of judgment; the AHP method does not take into account the uncertainty associated with the mapping of human judgment to a number by natural language; the ranking of the AHP method is rather imprecise; and the subjective judgment by perception, evaluation, improvement and selection based on preference of decision-makers have great influence on the AHP results [22].To overcome these problems, several researchers integrate fuzzy theory with AHP to improve the uncertainty and some researchers integrated fuzzy AHPwith other fuzzy methods such as fuzzy TOPSIS[23]-[25]. The main advantage of AHP and fuzzy AHPis hidden in this note that pairwise comparisons led to more convenient, realistic and logical appraisement of alternatives rather than other methods and techniques. This advantage of AHP and fuzzy AHP can led to more usability of them as core of model's evaluation. In along of it other methods can be used as core of ranking operation, so fuzzy AHP has a complementary role in model and is base of comparisons. 


\section{The integrated methodology}

Due to enrichment and improvement of pairwise comparisons in fuzzy AHP, we integrated the developed fuzzy VIKOR method after normalization in fuzzy AHP. Advantage of such integration is capability of dual- disciplinary of fuzzy VIKOR to fuzzy AHP. Inproposed method fuzzy VIKOR has been developed for situations that we face with both group utility and individual regret of the opponentsimultaneously. At this development fuzzy VIKOR appropriated at two calculations levels and one decision-making level. Cited method is able to apply to any MCDM with inconsistent judgment's conditions. At first level we calculate scores for evaluation's criterias that should be attainable all of them and then do calculations which related to sub-criterias that attainment of them individually can estimated main criterias. Fig. 2 illustrated a schematic step-by-step diagram of proposed methodology.The mathematical steps of proposed methodology explainedin appendix 1.

\subsection{Identification of necessary criterias and structuring of evaluation hierarchy}

In this step, with considering of business opportunities and determining of objective and strategies according to those opportunities, appropriated criterias and their sub-criterias have been selected and then decision hierarchy should be structured based on them. At this stage, it should be established two kind of mentionedcriterias. First those can be attainable with attainment of respective collection of their sub-criteriasand other,thosethat can be attainable with attainment of one or several sub-criterias. We referee to first category as "collectivecriterias"and next"Individual criterias". Also according to this step it's inevitable, Identification of potential partners and determining of appropriated and proposed alternatives. Tasks of this step should be adapted with different aspects of VE's life cycle.

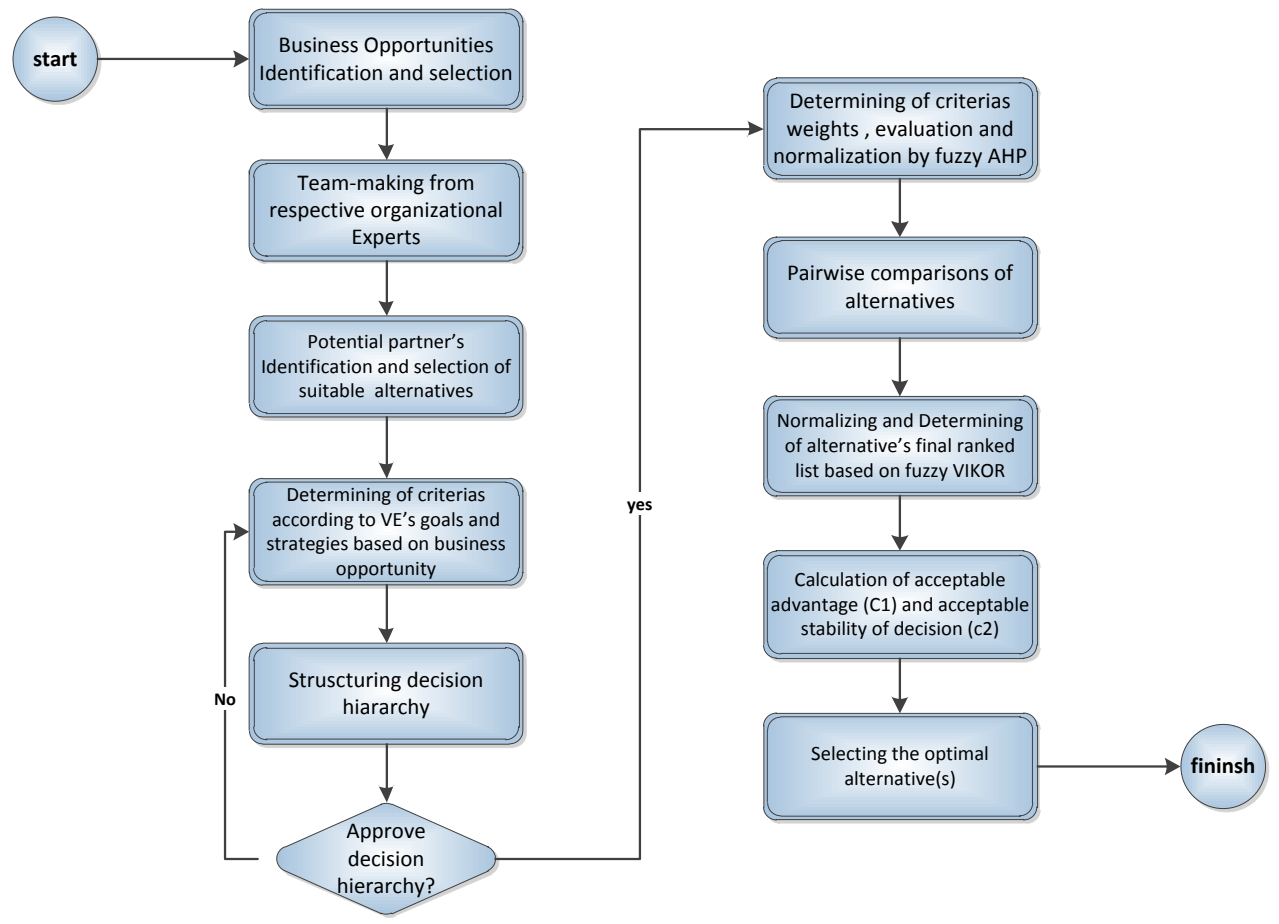

Figure 2. schematic diagram of proposed methodology 


\subsection{Determining of weight of hierarchy's dimensions}

In this step we need to define fuzzy number and determine linguistic variables for comparisons.A linguistic variable is a variable values of which are linguistic terms [26].Application of such concept, return to complex and uncertain situations which are ill-defined too. For examplechen and haung (1992) define the $(0.00,0.00,0.25)$ as fuzzy numbers for very low (VL) linguistic variable and so $(0.00,0.25,0.50)$ for low (L) linguistic variable and so on[27]. Some necessary definitions presented in appendix 1 .

These terms used as importance's comparisons of each criteriasrather than others. Thenfor each main criterias; matrixes of sub-criterias for pairwise-comparisons separatelyhave been established. Frist matrix of sub-criterias which has been established belonged to collective criterias and next to Individual criterias. Such as it mentioned above, with performing or attaining of each of Second categorized sub-criterias independently, respective main criterias can be attainable.Then we should complete pairwise-comparisons separately by respective sub-criterias using of fuzzy AHP technique. Also we should calculate weight of sub-criterias at pairwise approach. For all kind of main criterias, comparisons' matrixes with looking to Eqs. (6)-(9) should be determined and normalized (see appendix 1).

\subsection{Determining of comparisons between alternatives}

In this step linguistic variables for alternatives'evaluation should be determined. Then pairwisecomparisons of alternatives should beestablished and calculated separately on each subcriterias. Due to considering different decision-makers opinions, aggregation of votes needs to be run.

Next according to section of developed fuzzy VIKOR from proposed methodology, wedetermine fuzzy best value (FBV) and fuzzy worst value (FWV)separately on collective and individual main criterias.Against previous calculations that belonged to weight of decision-making structure, the normalization of matrixes inthis step performedby using fuzzy VIKOR methods.Then matrixes should benormalized and ranked-list extracted.

Note that, $v$ 'in Eq. (25) means the weight of the strategy of the maximum group utility. When $v^{\prime}>0.5$ the decision tends toward the maximum majority rule;and if $v^{\prime}<0.5$ the decision tends toward the individual regret of the opponent. We use $v^{\prime}>0.8$ because of here, we face with sub-criterias of collective main-criteria and in calculation of $v^{\prime \prime}$, we assign $v^{\prime \prime}<0.3$ to nature of its main-criteria. We referee to authority of strategic plan and objective whichorganization want to emphasize on individual main-criteria or collective main-criteria in its construct of appraisement. $w<0.5$ apply first strategy and $w>0.5$ emphasize on second strategy.

\subsection{Analyzing of results and final decision-making}

Finally,considering of decision-making status and typical evaluation of alternatives, we calculate final results, examine it according to Eqs. (28)-(31) and recognize optimal solution or solutions. Depended on acceptable advantage and acceptable stability of decision - C1 and C2; it may has unique optimal solution or not.The final result depended on experts' appraisements, typical main criterias, sub-criterias and organization attention to evaluation process as collective process or individual process.At end we report status of results and final decision.

\section{Case study}

Alpha system is a virtual enterprise with information, virtual-training and automation services. Some of its services including:

- Consultation about System analysis and design 
- Web-based training program

- Consultation about Business process reengineering on web-based services

- Web-based organizational Project handling and management

- Appraisement of web-based services' quality

- Consultation about ERP selection and implementation

- BPMS designing and planning in sector of public services

According to revised strategies, objective and goals by adopting it to new opportunities, enterprise decides to outsource section of web-based training in supporting of respective consultation. Top manager and change management believe outsourcing cited process led to cost optimization and focusing on main tasks and critical operations. Board of directors specifies five members of organizational experts as team of decision-makers for evaluation of alternatives. This process including several of sub-process and main aim of enterprise is performing of process as agile and speedy changeable services. From managers perspective agility factor and modularity have vital position in evaluation of alternatives. Second factor explained because of enterprise need to be compatible with environmental opportunities and have agile dissolution and reconfiguration. Fig. 3Depictscriterias and sub-criteriasthat are vital for alpha system's objectives and strategies.According to it, we establish hierarchy dimensions and determine weight of criterias.

Experts vote to equality of dual main criterias thus there is no need to matrix's establishment for pairwise comparisons of both mentioned main criterias-agility and modularity. First considering experts' knowledge, we define linguistic variables for evaluation process.Then pairwise comparisons of sub-criterias according to Eq. (6) and Eq. (9) performed. According to them we calculate $\mathrm{W}^{\prime}$ and $\mathrm{W}$ for sub-criterias in first and second main criteria and determine normalization Vector. The final results which showed in table 5 are aligned withsecond step of proposed methodology.

Then we acquire experts' votes about comparisons of alternatives base on sub-criteriasthat grouped in each main criteria then aggregate and calculate average of them. All of detailed calculations and respective tableswerepresented in appendix 2 .

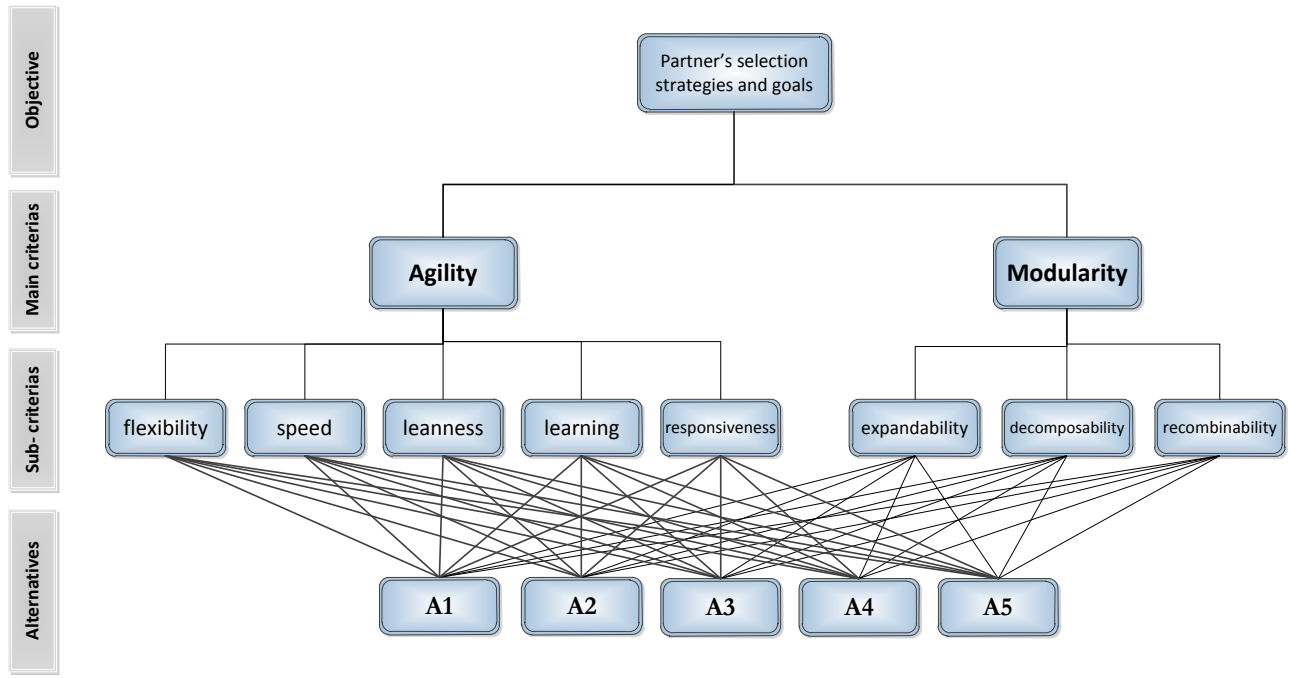

Figure. 3 The decision hierarchy of partner's selection of VE of alpha-system 
Table 5.The normalized weights of main-criteria and sub-criterias according to fuzzy AHP techniques

\begin{tabular}{|c|c|c|c|c|c|}
\hline \multirow{3}{*}{$\begin{array}{c}\text { Agility's } \\
\text { sub-criterias }\end{array}$} & SC1 & SC2 & SC3 & $\mathrm{SC} 4$ & SC5 \\
\hline & Flexibility & Speed & Leanness & Learning & Responsive \\
\hline & 0.62 & 0.37 & 0 & 0 & 0.01 \\
\hline \multirow{3}{*}{$\begin{array}{l}\text { Modularity's } \\
\text { sub-criterias }\end{array}$} & SC6 & SC7 & SC8 & & \\
\hline & Expandability & Decomposability & Recombinability & & \\
\hline & 0.34 & 0.52 & 0.14 & & \\
\hline
\end{tabular}

In the next step, we determine matrix of pairwise comparisons of alternatives for each subcriterias and perform related calculations (table 6-16).According to table 15, in Flexibility subcriteria, $S_{1}$ and $S_{4}$ are larger than other. With referee to table 14 , it has been seem that selection of $S_{4}$ is better decision. These results should be for each sub-criteria. Because of nature of alpha-system enterprise, we need to have one matrix for agility and one matrix for modularity. In the continuance of calculation we complete some remained cells of matrixes $\widetilde{C C}$ and $\widetilde{I C}$, determine the fuzzy best value (FBV) and fuzzy worst value (FWV). Then according to Eq. (19) and Eq. (20) we normalize mentioned matrixes based on typical main criterias- individual and collective criteria, separately. Note, the weights of sub-criterias calculated in previous pairwise comparisons using fuzzy AHP for main criterias and sub criterias, however expert cited same importance for both main criterias.

Table 17.fuzzy best value (FBV) and fuzzy worst value (FWV) per each criteria

\begin{tabular}{|l|c|c|c|c|c|c|}
\hline & \multicolumn{5}{|c|}{$\tilde{f}_{j^{*}}^{*}$} & \multicolumn{3}{|c|}{$\tilde{f}_{j^{\prime}}^{-}$} \\
\hline SC1 & 0.22 & 0.32 & 0.46 & 0.06 & 0.08 & 0.12 \\
\hline SC2 & 0.21 & 0.31 & 0.44 & 0.06 & 0.08 & 0.12 \\
\hline SC3 & 0.21 & 0.31 & 0.44 & 0.06 & 0.08 & 0.11 \\
\hline SC4 & 0.24 & 0.34 & 0.48 & 0.06 & 0.08 & 0.11 \\
\hline SC5 & 0.22 & 0.31 & 0.44 & 0.06 & 0.09 & 0.12 \\
\hline & \multicolumn{7}{|c|}{$\tilde{f}_{j^{\prime \prime}}^{*}$} \\
\hline SC6 & 0.21 & 0.30 & 0.43 & 0.07 & 0.09 & 0.15 \\
\hline SC7 & 0.22 & 0.33 & 0.51 & 0.07 & 0.11 & 0.18 \\
\hline SC8 & 0.20 & 0.30 & 0.44 & 0.07 & 0.09 & 0.14 \\
\hline
\end{tabular}

Table 18. Calculation of $S_{j}$ and $R_{\mathbf{j}}$ for whole of sub-criterias

\begin{tabular}{|c|c|c|c|c|c|c|c|c|c|c|c|c|c|c|c|c|}
\hline \multicolumn{17}{|c|}{ Agility - Collective main-criteria } \\
\hline & & \multicolumn{3}{|c|}{ A1 } & \multicolumn{3}{|c|}{ A2 } & \multicolumn{3}{|c|}{ A3 } & \multicolumn{3}{|c|}{ A4 } & \multicolumn{3}{|c|}{ A5 } \\
\hline \multirow{5}{*}{$\widetilde{C C}=$} & SC1 & 0.000 & $\begin{array}{c}0.00 \\
0\end{array}$ & $\begin{array}{c}0.00 \\
0\end{array}$ & $\begin{array}{c}0.07 \\
7\end{array}$ & $\begin{array}{c}0.09 \\
5\end{array}$ & $\begin{array}{c}0.10 \\
7\end{array}$ & $\begin{array}{c}0.45 \\
9\end{array}$ & $\begin{array}{c}0.43 \\
0\end{array}$ & $\begin{array}{c}0.41 \\
6\end{array}$ & $\begin{array}{c}0.40 \\
8\end{array}$ & $\begin{array}{c}0.41 \\
9\end{array}$ & $\begin{array}{c}0.42 \\
4\end{array}$ & $\begin{array}{c}0.62 \\
0\end{array}$ & $\begin{array}{c}0.62 \\
0\end{array}$ & $\begin{array}{c}0.62 \\
0\end{array}$ \\
\hline & SC2 & 0.000 & $\begin{array}{c}0.00 \\
0\end{array}$ & $\begin{array}{c}0.00 \\
0\end{array}$ & $\begin{array}{c}0.01 \\
7\end{array}$ & $\begin{array}{c}0.03 \\
4\end{array}$ & $\begin{array}{c}0.04 \\
5\end{array}$ & $\begin{array}{c}0.24 \\
6\end{array}$ & $\begin{array}{c}0.23 \\
5\end{array}$ & $\begin{array}{c}0.23 \\
1\end{array}$ & $\begin{array}{c}0.24 \\
2\end{array}$ & $\begin{array}{c}0.25 \\
2\end{array}$ & $\begin{array}{c}0.26 \\
1\end{array}$ & $\begin{array}{c}0.37 \\
0\end{array}$ & $\begin{array}{c}0.37 \\
0\end{array}$ & $\begin{array}{c}0.37 \\
0\end{array}$ \\
\hline & SC3 & 0.000 & $\begin{array}{c}0.00 \\
0 \\
\end{array}$ & $\begin{array}{c}0.00 \\
0\end{array}$ & $\begin{array}{c}0.00 \\
0 \\
\end{array}$ & $\begin{array}{c}0.00 \\
0 \\
\end{array}$ & $\begin{array}{c}0.00 \\
0\end{array}$ & $\begin{array}{c}0.00 \\
0\end{array}$ & $\begin{array}{c}0.00 \\
0\end{array}$ & $\begin{array}{c}0.00 \\
0\end{array}$ & $\begin{array}{c}0.00 \\
0\end{array}$ & $\begin{array}{c}0.00 \\
0 \\
\end{array}$ & $\begin{array}{c}0.00 \\
0\end{array}$ & $\begin{array}{c}0.00 \\
0 \\
\end{array}$ & $\begin{array}{c}0.00 \\
0 \\
\end{array}$ & $\begin{array}{c}0.00 \\
0\end{array}$ \\
\hline & SC4 & 0.000 & $\begin{array}{c}0.00 \\
0\end{array}$ & $\begin{array}{c}0.00 \\
0\end{array}$ & $\begin{array}{c}0.00 \\
0\end{array}$ & $\begin{array}{c}0.00 \\
0\end{array}$ & $\begin{array}{c}0.00 \\
0\end{array}$ & $\begin{array}{c}0.00 \\
0\end{array}$ & $\begin{array}{c}0.00 \\
0\end{array}$ & $\begin{array}{c}0.00 \\
0\end{array}$ & $\begin{array}{c}0.00 \\
0\end{array}$ & $\begin{array}{c}0.00 \\
0\end{array}$ & $\begin{array}{c}0.00 \\
0\end{array}$ & $\begin{array}{c}0.00 \\
0\end{array}$ & $\begin{array}{c}0.00 \\
0\end{array}$ & $\begin{array}{c}0.00 \\
0\end{array}$ \\
\hline & SC5 & 0.002 & $\begin{array}{c}0.00 \\
1 \\
\end{array}$ & $\begin{array}{c}0.00 \\
1 \\
\end{array}$ & $\begin{array}{c}0.00 \\
0 \\
\end{array}$ & $\begin{array}{c}0.00 \\
0\end{array}$ & $\begin{array}{c}0.00 \\
0 \\
\end{array}$ & $\begin{array}{c}0.00 \\
7 \\
\end{array}$ & $\begin{array}{c}0.00 \\
7\end{array}$ & $\begin{array}{c}0.00 \\
6 \\
\end{array}$ & $\begin{array}{c}0.00 \\
6 \\
\end{array}$ & $\begin{array}{c}0.00 \\
6 \\
\end{array}$ & $\begin{array}{c}0.00 \\
6 \\
\end{array}$ & $\begin{array}{c}0.01 \\
0 \\
\end{array}$ & $\begin{array}{c}0.01 \\
0 \\
\end{array}$ & $\begin{array}{c}0.01 \\
0 \\
\end{array}$ \\
\hline \multicolumn{2}{|c|}{$\tilde{S}_{j^{\prime}}$} & 0.00 & 0.00 & 0.00 & 0.00 & 0.09 & 0.13 & 0.15 & 0.71 & 0.67 & 0.65 & 0.66 & 0.68 & 0.69 & 1.00 & 1.00 \\
\hline \multicolumn{2}{|c|}{$\widetilde{\boldsymbol{R}}_{\boldsymbol{j}^{\prime}}$} & 0.002 & $\begin{array}{c}0.00 \\
2\end{array}$ & $\begin{array}{c}0.00 \\
1\end{array}$ & $\begin{array}{c}0.00 \\
1\end{array}$ & $\begin{array}{c}0.07 \\
7\end{array}$ & $\begin{array}{c}0.09 \\
5\end{array}$ & $\begin{array}{c}0.10 \\
7\end{array}$ & $\begin{array}{c}0.45 \\
9\end{array}$ & $\begin{array}{c}0.43 \\
0\end{array}$ & $\begin{array}{c}0.41 \\
6 \\
\end{array}$ & $\begin{array}{c}0.40 \\
8 \\
\end{array}$ & $\begin{array}{c}0.41 \\
9 \\
\end{array}$ & $\begin{array}{c}0.42 \\
4\end{array}$ & $\begin{array}{c}0.62 \\
0\end{array}$ & $\begin{array}{c}0.62 \\
0\end{array}$ \\
\hline \multicolumn{17}{|c|}{ Modularity - Individual main-criteria } \\
\hline \multirow{2}{*}{$\widetilde{I C}=$} & SC6 & 0.089 & $\begin{array}{c}0.05 \\
3\end{array}$ & $\begin{array}{c}0.03 \\
0\end{array}$ & $\begin{array}{c}0.00 \\
0\end{array}$ & $\begin{array}{c}0.00 \\
0\end{array}$ & $\begin{array}{c}0.00 \\
0\end{array}$ & $\begin{array}{c}0.24 \\
7\end{array}$ & $\begin{array}{c}0.22 \\
6\end{array}$ & $\begin{array}{c}0.21 \\
9\end{array}$ & $\begin{array}{c}0.21 \\
5\end{array}$ & $\begin{array}{c}0.21 \\
9\end{array}$ & $\begin{array}{c}0.22 \\
5\end{array}$ & $\begin{array}{c}0.34 \\
0\end{array}$ & $\begin{array}{c}0.34 \\
0\end{array}$ & $\begin{array}{c}0.34 \\
0\end{array}$ \\
\hline & SC7 & 0.365 & $\begin{array}{c}0.28 \\
1\end{array}$ & $\begin{array}{c}0.25 \\
2 \\
\end{array}$ & $\begin{array}{c}0.00 \\
0 \\
\end{array}$ & $\begin{array}{c}0.00 \\
0 \\
\end{array}$ & $\begin{array}{c}0.00 \\
0\end{array}$ & $\begin{array}{c}0.41 \\
1 \\
\end{array}$ & $\begin{array}{c}0.37 \\
8 \\
\end{array}$ & $\begin{array}{c}0.37 \\
6\end{array}$ & $\begin{array}{c}0.33 \\
1 \\
\end{array}$ & $\begin{array}{c}0.33 \\
9 \\
\end{array}$ & $\begin{array}{c}0.35 \\
6\end{array}$ & $\begin{array}{c}0.52 \\
0 \\
\end{array}$ & $\begin{array}{c}0.52 \\
0 \\
\end{array}$ & $\begin{array}{c}0.52 \\
0 \\
\end{array}$ \\
\hline
\end{tabular}




\begin{tabular}{|c|c|c|c|c|c|c|c|c|c|c|c|c|c|c|c|}
\hline SC8 & 0.008 & $\begin{array}{c}0.00 \\
0 \\
\end{array}$ & $\begin{array}{c}0.00 \\
0 \\
\end{array}$ & $\begin{array}{c}0.00 \\
0 \\
\end{array}$ & $\begin{array}{c}0.00 \\
2 \\
\end{array}$ & $\begin{array}{c}0.00 \\
9 \\
\end{array}$ & $\begin{array}{c}0.10 \\
7 \\
\end{array}$ & $\begin{array}{c}0.09 \\
8 \\
\end{array}$ & $\begin{array}{c}0.09 \\
7 \\
\end{array}$ & $\begin{array}{c}0.08 \\
7 \\
\end{array}$ & $\begin{array}{c}0.09 \\
0 \\
\end{array}$ & $\begin{array}{c}0.09 \\
4 \\
\end{array}$ & $\begin{array}{c}0.14 \\
0 \\
\end{array}$ & $\begin{array}{c}0.14 \\
0 \\
\end{array}$ & $\begin{array}{c}0.14 \\
0\end{array}$ \\
\hline$\tilde{\boldsymbol{S}}_{\boldsymbol{j}}{ }^{\prime \prime}$ & 0.463 & $\begin{array}{c}0.46 \\
3\end{array}$ & $\begin{array}{c}0.33 \\
4\end{array}$ & $\begin{array}{c}0.28 \\
2\end{array}$ & $\begin{array}{c}0.00 \\
0\end{array}$ & $\begin{array}{c}0.00 \\
2\end{array}$ & $\begin{array}{c}0.00 \\
9\end{array}$ & $\begin{array}{c}0.76 \\
5\end{array}$ & $\begin{array}{c}0.70 \\
3\end{array}$ & $\begin{array}{c}0.69 \\
2\end{array}$ & $\begin{array}{c}0.63 \\
4\end{array}$ & $\begin{array}{c}0.64 \\
8\end{array}$ & $\begin{array}{c}0.67 \\
5\end{array}$ & $\begin{array}{c}1.00 \\
0\end{array}$ & $\begin{array}{c}1.00 \\
0\end{array}$ \\
\hline$\widetilde{\boldsymbol{R}}_{\boldsymbol{j}}^{\prime \prime}$ & 0.365 & $\begin{array}{c}0.36 \\
5\end{array}$ & $\begin{array}{c}0.28 \\
1\end{array}$ & $\begin{array}{c}0.25 \\
2 \\
\end{array}$ & $\begin{array}{c}0.00 \\
0\end{array}$ & $\begin{array}{c}0.00 \\
2\end{array}$ & $\begin{array}{c}0.00 \\
9\end{array}$ & $\begin{array}{c}0.41 \\
1\end{array}$ & $\begin{array}{c}0.37 \\
8\end{array}$ & $\begin{array}{c}0.37 \\
6\end{array}$ & $\begin{array}{c}0.33 \\
1\end{array}$ & $\begin{array}{c}0.33 \\
9 \\
\end{array}$ & $\begin{array}{c}0.35 \\
6\end{array}$ & $\begin{array}{c}0.52 \\
0\end{array}$ & $\begin{array}{c}0.52 \\
0\end{array}$ \\
\hline
\end{tabular}

According to Eqs.(21)-(27) and considering of Enterprise's viewpoint, We assume v'=0.95 and $v^{\prime \prime}=0.1$ to have large appropriated effects on each stage including calculation of both collective and Individual sub-criterias. $\mathrm{w}=0.4$, this means organization decides to emphasize on individual sub-criteriasin its Appraisement's structure.

Table 19. Calculation of $S_{j}^{*}, S_{j}^{-}, R_{j}^{*}$ and $R_{j}$ for each of main-criterias

\begin{tabular}{|c|c|c|c|c|c|}
\hline \multicolumn{6}{|c|}{$\begin{array}{l}\text { The first section of decision-making } \\
\text { (Agility: only collective main-criteria) }\end{array}$} \\
\hline 0.00 & 0.00 & 0.00 & 1.00 & 1.00 & 1.00 \\
\hline 0.00 & 0.00 & 0.00 & 0.62 & 0.62 & 0.62 \\
\hline \multicolumn{6}{|c|}{$\begin{array}{l}\text { The second section of decision-making } \\
\text { (Modularity: only Individual main-criteria) }\end{array}$} \\
\hline 0.00 & 0.00 & 0.01 & 1.00 & 1.00 & 1.00 \\
\hline 0.00 & 0.00 & 0.01 & 0.52 & 0.52 & 0.52 \\
\hline
\end{tabular}

Table 20. Final calculation and rancked list of alternatives

\begin{tabular}{|c|c|c|c|c|c|c|c|c|c|c|c|c|c|c|c|}
\hline & \multicolumn{3}{|c|}{$\mathbf{A}_{1}$} & \multicolumn{3}{|c|}{$\mathbf{A}_{2}$} & \multicolumn{3}{|c|}{$\mathbf{A}_{3}$} & \multicolumn{3}{|c|}{$\mathbf{A}_{4}$} & \multicolumn{3}{|c|}{$\mathbf{A}_{5}$} \\
\hline$\tilde{Q}_{i^{\prime}}$ & $\begin{array}{c}0.00 \\
0 \\
\end{array}$ & $\begin{array}{c}0.00 \\
0 \\
\end{array}$ & $\begin{array}{c}0.00 \\
0\end{array}$ & $\begin{array}{c}0.09 \\
3 \\
\end{array}$ & $\begin{array}{c}0.12 \\
9 \\
\end{array}$ & $\begin{array}{c}0.15 \\
3 \\
\end{array}$ & $\begin{array}{c}0.71 \\
3 \\
\end{array}$ & $\begin{array}{c}0.67 \\
2 \\
\end{array}$ & $\begin{array}{c}0.65 \\
4 \\
\end{array}$ & $\begin{array}{c}0.65 \\
6\end{array}$ & $\begin{array}{c}0.67 \\
7 \\
\end{array}$ & $\begin{array}{c}0.69 \\
0 \\
\end{array}$ & $\begin{array}{c}1.00 \\
0 \\
\end{array}$ & $\begin{array}{c}1.00 \\
0 \\
\end{array}$ & $\begin{array}{c}1.00 \\
0 \\
\end{array}$ \\
\hline $\mathrm{Q}_{\mathrm{i}^{\prime}}$ & \multicolumn{3}{|c|}{0.000} & \multicolumn{3}{|c|}{0.125} & \multicolumn{3}{|c|}{0.680} & \multicolumn{3}{|c|}{0.674} & \multicolumn{3}{|c|}{1.000} \\
\hline$\tilde{Q}_{i^{\prime \prime}}$ & $\begin{array}{c}0.67 \\
8\end{array}$ & $\begin{array}{c}0.51 \\
8\end{array}$ & $\begin{array}{c}0.45 \\
5\end{array}$ & $\begin{array}{c}0.00 \\
0\end{array}$ & $\begin{array}{c}0.00 \\
0\end{array}$ & $\begin{array}{c}0.00 \\
0\end{array}$ & $\begin{array}{c}0.78 \\
8\end{array}$ & $\begin{array}{c}0.72 \\
4\end{array}$ & $\begin{array}{c}0.71 \\
5\end{array}$ & $\begin{array}{c}0.63 \\
7\end{array}$ & $\begin{array}{c}0.65 \\
0\end{array}$ & $\begin{array}{c}0.67 \\
7\end{array}$ & $\begin{array}{c}1.00 \\
0\end{array}$ & $\begin{array}{c}1.00 \\
0\end{array}$ & $\begin{array}{c}1.00 \\
0\end{array}$ \\
\hline$Q_{i "}$ & \multicolumn{3}{|c|}{0.551} & \multicolumn{3}{|c|}{0.000} & \multicolumn{3}{|c|}{0.743} & \multicolumn{3}{|c|}{0.655} & \multicolumn{3}{|c|}{1.000} \\
\hline$\tilde{Q}_{i}$ & \multicolumn{3}{|c|}{0.330} & \multicolumn{3}{|c|}{0.050} & \multicolumn{3}{|c|}{0.717} & \multicolumn{3}{|c|}{0.662} & \multicolumn{3}{|c|}{1.000} \\
\hline Rank* $^{*}$ & \multicolumn{3}{|c|}{2} & \multicolumn{3}{|c|}{$1^{*}$} & \multicolumn{3}{|c|}{4} & \multicolumn{3}{|c|}{3} & \multicolumn{3}{|c|}{5} \\
\hline
\end{tabular}

According to table $20, \mathrm{~A}_{2}$ and $\mathrm{A}_{1}$ are best solutions. With attention of $\mathrm{C} 1$ and $\mathrm{C} 2$; $\mathrm{C} 1$ is acceptable but $\mathrm{C} 2$ or acceptable stability of decision isn't acceptable. However acceptable advantage of selection of $A_{2}$ against $A_{1}$ In result $A_{1}$ and $A_{2}$ are same.

\section{Conclusion}

In this paper we combine Fuzzy AHP method with developed Fuzzy VIKOR. The comparative advantage of proposed model from one viewpoint related to simplifying comparisons of alternatives with pairwise comparisons, and from other viewpointrelated to authority of model to satisfy group utility and individual regret of the opponentsimultaneously in context of VEs. Then model apply to one VE which have willing to outsource their process for guarantee of agility and synergic responsiveness to changing demand at whole of Enterprise's supply chain. Proposed model can be apply to extended domain of appraisement and judgments' activities. The main goal that satisfied by this model, is capability of Multicriteria decision-making in complex and heterogeneous context; 
situations which have incongruent nature that affects appraisement structure and decision-making process.

\section{References}

[1] Camarinha-Matos, L.M., Afsarmanesh, H., Garita, C., and Lima, C., "Towards an architecture for virtual enterprises", Journal of Intelligent Manufacturing, Vol. 9, No.2, pp. 189-199, 1998.

[2] Gazendam, H.W.M., "Semiotics, Virtual Organisations, and Information Systems", In Liu, K., Clarke, R., Anderson, P.B., and Stamper, R.K. (eds.), Information, Organisation and Technology: Studies in Organisational Semiotics", Boston: Kluwer Academic Publishers, pp. 1- 48, 2001.

[3] Putnik, G.D., Cunha, M.M., Sousa, R., and Avila, P., "Virtual Enterprise Integration: Challenges of a New Paradigm", In Cunha, M.M., Putnik, G. (eds.), "Virtual Enterprise Integration: Technological and Organizational Perspectives", Idea group inc., Hershey, 2005.

[4] Sharifi, H., and Zhang, Z., "A methodology for achieving agility in manufacturing organization: An introduction", International Journal of Production Economics, Vol. 62, pp. 7-22, 1999.

[5] Yusuf, Y.Y., Sarhadi, M., and Gunasekaran, A., "Agile manufacturing: The drivers, concepts and attributes", International Journal of Production Economic, Vol. 62, pp. 33-43, 1999.

[6] Goldman, S.L., Nagel, R.N., and Preiss, K., "Agile Competitors and Virtual Organizations: Strategies for Enriching the Customer", Van Nostrand Reinhold, New York, 1995.

[7] Kidd, P.T., "Agile Manufacturing: Forging New Frontiers", Addison-Wesley, London, 1994

[8] Sharifi, H., and Zhang, Z., "Agile manufacturing in practice-application of a methodology", International Journal of Operations and Production Management, Vol. 21, pp. 772-794, 2001.

[9] Kim, T-Y., Lee, S., Kim, K., and Kim, C-H., and, "A modeling framework for agile and interoperable virtual enterprises", Computers in Industry, Vol. 57, pp. 204-217, 2006.

[10] Anderson, D.M., "Agile Product Development For Mass Customization: How to Develop and Deliver Products for Mass Customization, Niche Markets, JIT, Build-To-Order and Flexible Manufacturing ", Irwin Professional Pub, Chicago, 1997

[11] Lau, A.K.W., Yam, R. C.M. , and Tang, E.P.Y., "Supply chain product co-development, product modularity and product performance ; Empirical evidence from Hong Kong manufacturers", Industrial Management \& Data Systems, Vol. 107, pp. 1036-1065, 2007.

[12] Fine, C.H.,"Clockspeed: Winning Industry Control in the Age of Temporary Advantage",Perseus Books, 1999

[13] Corvello, V., and Migliarese, P., "Virtual forms for the organization of production: A comparative analysis", International Journal of Production Economics, Vol. 110, pp. 5-15, 2007.

[14] Baldwin, C.Y., and Clark, K.B., "Managing in an Age of Modularity", Harvard Business Review, Vol. 75, No. 5, pp. 84-93, 1997.

[15] Towill, D.R. , "The seamless supply chain - the predators strategic advantage", International Journal of the Techniques of Manufacturing, Vol. 13, No. 1, pp. 37-56, 1997.

[16] Aissaoui, N., Haouari, H., and Elkafi, H., "Supplier selection and order lot sizing modeling: A review", Computers and Operations Research, Vol. 34, pp. 3516-3540, 2007

[17] Qumer, A., and Henderson-Sellers, B., "Measuring agility and adaptability of agile methods: a 4Dimensional Analytical Tool", Proceeding of IADIS International Conference Applied Computing, IADIS Press, pp. 503-507, 2006

[18] Schilling, M.A., "Modularity in multiple disciplines", In Guard,R., Kumaraswamy, A., and Langlois, R. (eds.), "Managing in the Modular Age: Architectures, Networks and Organizations", pp. 203-214, Oxford, Blackwell Publishers, England, 2003

[19] Opricovic, S., "Multicriteria optimization of civil engineering systems", Faculty of Civil Engineering, Belgrade, 1998

[20] Opricovic, S., and Tzeng, G-H., "Compromise solution by MCDM methods: A comparative analysis of VIKOR and TOPSIS", European Journal of Operational Research, Vol. 156, pp. 445-455, 2004.

[21] Saaty, T.L., "The Analytic Hierarchy Process", McGraw-Hill, New York, 1980. 
Peyman mohammady, Amin Amid / TJMCS Vol .1 No.4 (2010) 413-434

[22] Yang, C-C., and Chen, B-S., "Key quality performance evaluation using Fuzzy AHP", Journal of the Chinese Institute of Industrial Engineers, Vol. 21, pp. 543-550, 2004.

[23] Sun, C-C., "A performance evaluation model by integrating fuzzy AHP and fuzzy TOPSIS methods", Expert Systems with Applications, Vol. 37, pp. 7745-7754, 2010

[24] Torfi, F., Z.Farahani, R., and Rezapour, S., "Fuzzy AHP to determine the relative weights of evaluation criteria and Fuzzy TOPSIS to rank the alternatives", Applied Soft Computing, Vol. 10, pp. 520-528, 2010

[25] Wang, J., Fan, K., and Wang, W., "Integration of fuzzy AHP and FPP with TOPSIS methodology for aeroengine health assessment", Expert Systems with Applications, Vol. 37, pp. 8516-8526, 2010

[26] Zadeh, L.A., "The concept of a linguistic variable and its application to approximate reasoning", Information science, Vol. 8, pp. 199-249, 1975

[27] Chen, S.J., and Huang, G.H., "Fuzzy Multiple Attribute Decision Making : Methods and Applications ", Springer, New York, 1992 


\section{Appendix1.Proposed methodology}

\section{Primary definitions}

We describe some of fuzzy sets and fuzzy mathematics'definitionsbriefly,thenpropose mathematical steps of proposed methodology below:

Definition 1.A fuzzy Set $\tilde{A}$ in a universe of discourse $\mathrm{X}$ is characterized by a membership function $f_{\tilde{A}}(x)$ which associates with each element $x$ in $\mathrm{X}$, a real number in the interval $[0,1]$. The function value $f_{\tilde{A}}(x)$ is termed as grade of membership of $x$ in $\tilde{A}$. The current study employ triangular form of fuzzy number that defined by a triple $(\mathrm{l}, \mathrm{m}, \mathrm{u})$. The membership function $f_{\tilde{A}}(x)$ defined as shown in Eq. (1). The diagram of such membership function illustrated in Fig. 1.

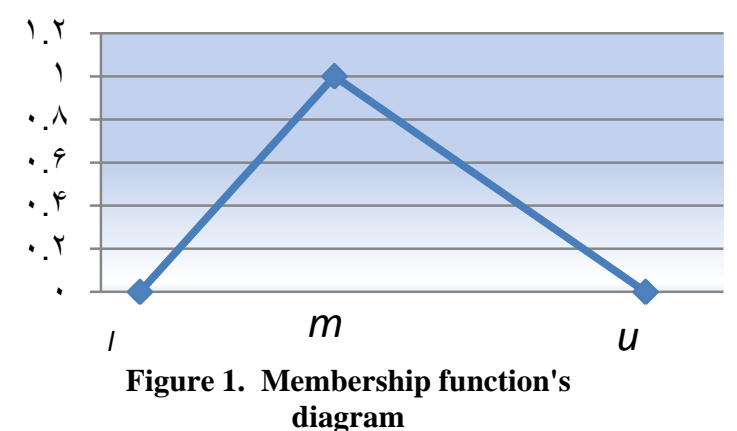

$f_{\tilde{A}}(x)=\left\{\begin{array}{cc}0 & , \quad x<l \\ \frac{x_{-} l}{m_{-} l} & , l \leq x \leq m \\ \frac{u-x}{u_{-} m}, & m \leq x \leq u\end{array}\right.$

Assume $\tilde{A}\left(l_{1}, m_{1}, u_{1}\right)$ and $\tilde{B}\left(l_{2}, m_{2}, u_{2}\right)$ are two triangular fuzzy numbers. Operational laws of these two triangular fuzzy numbers shown as follow:

$\tilde{A}(+) \tilde{B}=\left(l_{1}+l_{2}, m_{1}+m_{2}, u_{1}+u_{2}\right)$

$\tilde{A}(-) \tilde{B}=\left(l_{1}-u_{2}, m_{1}-m_{2}, u_{1}-l_{2}\right)$

$\tilde{A}(\times) \tilde{B}=\left(l_{1} \cdot l_{2}, m_{1} \cdot m_{2}, u_{1} \cdot u_{2}\right)$

$\tilde{A}(/) \tilde{B}=\left(l_{1} / u_{2}, m_{1} / m_{2}, u_{1} / l_{2}\right)$

Forl $l_{1}, m_{1}, u_{1}, l_{2}, m_{2}, u_{2}>0$

Definition 2.A linguistic variable is a variable values of which are linguistic terms. The application of such concept returns to complex and uncertain situations which are ill-defined too. For example the following fuzzy numbers defined by chen and haung (1992).

Very Low $(\mathrm{VL})=(0.00,0.00,0.25)$

$\operatorname{Low}(\mathrm{L})=(0.00,0.25,0.50)$

Medium(M) $=(0.25,0.50,0.75)$

$\operatorname{High}(\mathrm{H})=(0.50,0.75,1.00)$ 
Very High $(\mathrm{VH})=(0.75,1.00,1.00)$

\section{Step1. Determining of hierarchy's dimensions}

Suppose following Variables:

m: number of alternatives.

$\mathrm{n}$ : number of decision-makers(experts team)

$k_{\mathrm{j}^{\prime}}$ : number of collective's sub-criteriasof $\mathrm{j}$ ' 'th criteria.

$l_{j}$ ": number of individual's sub-criteriasof $\mathrm{j}$ "' 'thcriteria.

Due to simplify calculations and representation of equations and operations, we assume that model have one main-criteria of collective criteria and one main-criteria of individual criteria( $j^{\prime}=j^{\prime \prime}=1$ ). Extension of operations to each main-criteria is simple. So we repeated Eq. (6) to Eq. (7) for each main criterias. We establish $\widetilde{C C}$ and $\widetilde{I C}$ matrixes. First matrix should be established for collective main criterias and next for individual main criterias. The concepts and different between have been described in paper, previous.

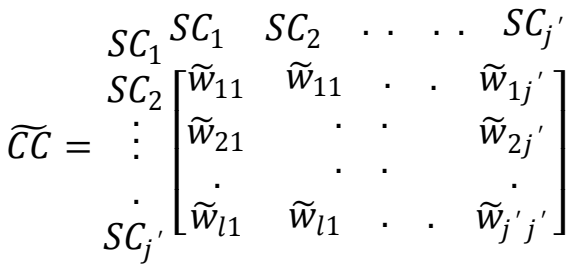

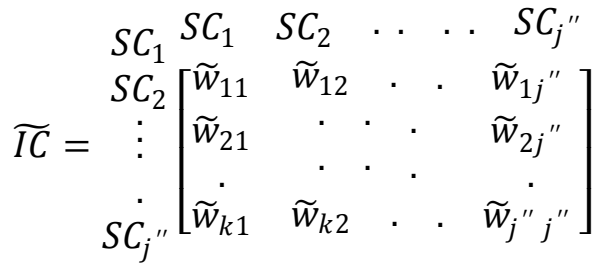

Where $\widetilde{w}_{j i}=\left(\widetilde{w}_{i j}\right)^{-1}=\left(\frac{1}{u_{i j}}, \frac{1}{m_{i j}}, \frac{1}{l_{i j}}\right)$

Weight of pairwise comparisons of criterias are mean of weight which experts-decisionmakersassigned them to each comparisons by linguistic variables and should be transformed to fuzzy triangular numbers.

$\widetilde{w}_{i j}=1 / n\left[\sum_{r=1}^{n} \widetilde{w}_{i j r}\right]$

Where $\mathrm{r}=1,2, \ldots, \mathrm{n}$;

For collective sub-criterias; $j=j^{\prime}=1,2, \ldots, k$ and

For individual sub-criterias; $j=j "=1,2, \ldots, l$

Here $\widetilde{w}_{i j}$ is weight of sub criteria $i$ against sub criteria $j$. First, these weightsassigned by experts (decision-makers) to each comparisons using linguistic variables and then those should be 
transformed to fuzzy triangular numbers or other form of fuzzy numbers. In continuance, pairwise comparisons should be calculated separately for each sub-criteriasbased on fuzzy AHP.

In this stage opinions about comparisons need to be aggregated and averaged and thenSC $\mathrm{j}_{j}$ calculated for all of them (sub-criterias) in class of main criterias (see Eq. (10)).The degree of possibility of $S C_{1}\left(l_{1}, m_{1}, u_{1}\right) \geq S C_{2}\left(l_{2}, m_{2}, u_{2}\right)$ define as indicated in Eq. (11). This equationDepictspossibility's degree of largeness of $\mathrm{SC}_{1}$ rather than $\mathrm{SC}_{2}$. These calculation apply for sub-criterias of individual-main criteria -IC matrix(es), second Section of previous appraisement.

$$
\begin{aligned}
& S C_{1}\left(l_{1}, m_{1}, u_{1}\right)=\left(\sum_{i=1}^{k} l_{1 i}, \sum_{i=1}^{k} m_{1 i}, \sum_{i=1}^{l} u_{1 i}\right) *\left(\frac{1}{\sum_{i=1}^{l} u_{1 i}}, 1 / \sum_{i=1}^{k} m_{1 i}, 1 / \sum_{i=1}^{k} u_{1 i}\right) \\
& V\left(S C_{1} \geq S C_{2}\right)=\left\{\begin{array}{c}
0, \quad l_{2} \geq u_{1} \\
\frac{u_{1}-l_{2},}{\left(u_{1}-l_{2}\right)+\left(m_{2}-m_{1}\right)} \quad, \quad \text { otherwise } \\
1 \quad, \quad m_{1} \geq m_{2}
\end{array}\right.
\end{aligned}
$$

To be able for comparing degree of largeness of criterias or sub-criterias than all of other criterias or sub-criterias for example for $\mathrm{SC}_{1}$ 's calculation, we need to calculate both $V\left(S C_{1} \geq S C_{j}\right)$ and $V\left(S C_{j} \geq S C_{1}\right)$ synchronously as depicted in Eq. (12).

$$
\begin{aligned}
& V\left(S C_{1} \geq S C_{j}\right)=V\left(S C_{1} \geq S C_{2}, \ldots, S C_{j}\right)=\min V\left(S C_{1} \geq S C_{j}\right) \\
& \text { Where } \mathrm{j} \neq 1 \text { and } \\
& \text { For collective sub-criterias } j=j^{\prime}=1,2, \ldots, k ; \text { and } \\
& \text { For individual sub-criterias } j=j^{\prime \prime}=1,2, \ldots, l
\end{aligned}
$$

Next matrixes of sub-criterias' weights as shown in Eq. (14) and Eq. (15)should be normalized. Note, normalization of refereed matrixes in this step are related to sub criteriasof decision-making structure and should be calculated using fuzzy AHP.

$$
\begin{aligned}
& W^{\prime}=\left(V\left(S C_{1} \geq S C_{j}\right), V\left(S C_{2} \geq S C_{j}\right), \ldots, V\left(S C_{k} \geq S C_{j}\right)\right)^{t} \\
& \text { Where } \\
& \text { for collective sub-criterias } j=j^{\prime}=1,2, \ldots, k ; \text { and } \\
& \quad \text { for individual sub-criterias } j=j^{\prime \prime}=1,2, \ldots, l
\end{aligned}
$$$$
W=\frac{w^{\prime}}{\sum w^{\prime}}
$$

These final weights are used in second construct of model- developed fuzzy VIKOR method as shown in Eq. (19) and Eq.(20).

\section{Step2. Determining of alternatives' comparisons}

2.1. Separately on each sub criterias, we establish matrixes of alternatives. The mentioned matrix should be established according to Eq. (6) or Eq. (7) with rows and columns which consisted of alternatives instead of subcriterias.Then,Eqs.(9)-(12) for alternatives' comparisons calculated.We prepare matrixes for applying developed and extended fuzzy VIKOR method. This revised methods 
described briefly below. Note normalizationof mentioned matrixes should be performedby using fuzzy VIKOR.

For describing of developed fuzzy VIKOR in this step, suppose Xij is

$$
\widetilde{\boldsymbol{D}}=\begin{array}{cccc}
\mathrm{A}_{1} & \mathrm{~A}_{2} \ldots & & \\
\\
\mathrm{A}_{1} \\
\mathrm{~A}_{2} \\
\vdots \\
\mathrm{A}_{\mathrm{m}}
\end{array}\left[\begin{array}{ccccc}
(1,1,1) & \tilde{x}_{12} & \ldots & \tilde{x}_{1 k} \\
\tilde{x}_{21} & (1,1,1) & \ldots & \tilde{x}_{2 k} \\
\vdots & \vdots & \ddots & \vdots \\
\tilde{x}_{m 1} & \tilde{x}_{m 2} & \ldots & (1,1,1)
\end{array}\right]
$$

Where $i=1,2, . ., m$

for collective sub-criterias $j=j^{\prime}=1,2, \ldots, k$;

for individual sub-criterias $j=j "=1,2, \ldots, l$;

Here, $x_{\mathrm{ij}}$ is rating of alternative $i$ against alternative $j$ respect to each criteria. These variables are same to $w_{\mathrm{ij}}$ in Eq. (8) so, we summarize some matrixes and calculations below.

2.2. Determine the fuzzy best value (FBV) and fuzzy worst value (FWV) for collective and individual main-criterias separately:

$\tilde{f}_{j}^{*}=\max \tilde{x}_{i j}$
$\tilde{f}_{j}^{-}=\min _{i} \tilde{x}_{i j}$

Where

$i=1,2, \ldots m$

for collective sub-criterias $j=j^{\prime}=1,2, \ldots, k$;

for individual sub-criterias $j=j^{\prime \prime}=1,2, \ldots, l$;

The points of combination of fuzzy AHP and fuzzy VIKOR in proposed method occur at two aspects. First aspect is that, the weights of sub criterias as shown in Eqs. (6)-(16)should be calculated using fuzzy AHP and next be used as weights of Eq. (19) and Eq. (20) in fuzzy VIKOR method. Second and most important aspect belonged to here Eq. (17) and Eq.(18); our criteria for recognition of minimum and maximum of $\tilde{x}_{i j}$. For each of comparisons in alternatives' matrixes, we calculate Eqs. (10)-(12)Separately on sub criterias. Then we determine minimum and maximum of $\tilde{x}_{i j}$ with considering of $S_{i}$ andSC $C_{i}$.In other hand, the probability's degree of largeness (PDL) of each of sub criterias against others; is most important criteria for recognition of minimum and maximum of $\tilde{x}_{i j}$. For example minimum of $\tilde{x}_{i j}$ respect to alternative which has smallest PDL and so on.

2.3. We determine normalized values of pervious matrixes according to Eq. (19) and Eq. (20) for each of alternatives. Note that Values of Eq. (19) and Eq. (20) should be calculated upon to collective and individual main-criterias separately.As mentioned, we extract weights of subcriteriasaccording to Eq. (15). Where $\tilde{S}_{i}$ is $A_{i}$ with respect to all criteria calculated by the sum of the distance for the FBV, and $\widetilde{R}_{i}$ is $A_{i}$ with respect to the j'th criteria calculated by the maximum distance 
of FBV. In Eq. (21) to Eq. (25) we separate equation depend on j' -collective criteria and j"'Individual criteria.

$\tilde{S}_{i}=\sum_{j=1}^{k \text { or } l} \widetilde{w}_{j}\left(\frac{\tilde{f}_{j}^{*}-\tilde{x}_{i j}}{\tilde{f}_{j}^{*}-\tilde{f}_{j}^{-}}\right)$

$\tilde{R}_{i}=\underset{j}{\max }\left[\widetilde{w}_{j}^{\prime \prime} \frac{\tilde{f}_{j}^{*}-\tilde{x}_{i j}}{\tilde{f}_{j}^{*}-\tilde{f}_{j}^{-}}\right]$

Where

$\mathrm{i}=1,2, \ldots \mathrm{m}$ for collective sub-criterias $\mathrm{j}=\mathrm{j} '=1,2, \ldots, \mathrm{k}$;

and for individual sub-criterias $\mathrm{j}=\mathrm{j} "=1,2, \ldots, \mathrm{l}$;

$\tilde{S}_{i^{\prime}}{ }^{*}=\min _{i} \tilde{S}_{i^{\prime}}$

$\tilde{S}_{i^{\prime}}^{-}=\underset{i}{\max } \tilde{S}_{i^{\prime}}$

$\widetilde{R}_{i^{\prime}}{ }^{*}=\min \widetilde{R}_{i^{\prime}}$

$\tilde{R}_{i^{\prime}}{ }^{-}=\max \widetilde{R}_{i^{\prime}}$

$i$

Here $\mathrm{S}_{\mathrm{j}}$ is the minimum value of $\tilde{S}_{i}$, which is the maximum majority rule or maximum group utility, and $\mathrm{R}_{\mathrm{j}}^{*}$ is the minimum value of $\tilde{R}_{i}$, which is the minimum individual regret of the opponent. Thus, the index $\tilde{Q}_{i}$ is obtained and is based on the consideration of both the group utility and individual regret of the opponent.

2.4. Determine values of $\widetilde{Q}_{i}$, sort them in increasing order and rank them from smaller to larger.

$\tilde{Q}_{i^{\prime}}=v^{\prime}\left[\frac{\tilde{S}_{i^{\prime}}-\tilde{S}_{i}{ }^{*}}{\tilde{S}_{i^{\prime}}{ }^{-}-\tilde{S}_{i^{\prime}}{ }^{*}}\right]+\left(1-v^{\prime}\right)\left[\frac{\tilde{R}_{i}{ }^{\prime}-\tilde{R}_{i^{\prime}}{ }^{*}}{\tilde{R}_{i^{\prime}}{ }^{-}-\tilde{R}_{i^{\prime}}{ }^{*}}\right]$

$\tilde{Q}_{i^{\prime \prime}}=v^{\prime \prime}\left[\frac{\tilde{S}_{i}{ }^{\prime \prime}-\tilde{S}_{i}{ }^{*}}{\tilde{S}_{i^{\prime \prime}}{ }^{-}-\tilde{S}_{i^{\prime \prime}}{ }^{*}}\right]+\left(1-v^{\prime \prime}\right)\left[\frac{\tilde{R}_{i}{ }^{\prime \prime}-\tilde{R}_{i}{ }^{*}}{\tilde{R}_{i^{\prime \prime}}{ }^{-}-\tilde{R}_{i^{\prime \prime}}{ }^{*}}\right]$

$\tilde{Q}_{i}=\omega * \tilde{Q}_{i^{\prime}}+(1-\omega) * \tilde{Q}_{i^{\prime \prime}}$

\section{Step3. Analyzing of calculated results}


In the last stepdepended to values of acceptable advantage and acceptable stability of decision $\mathrm{C} 1$ and $\mathrm{C} 2$ - we determine final decision as optimal solution for evaluation. Assume $\mathrm{A}_{1}$ is first optimal solution and $\mathrm{A}_{2}$ is second.

$\left[\right.$ C1]: $Q\left(A_{2}\right)-Q\left(A_{1}\right) \geq D Q$

$D Q=\frac{1}{m-1}(I F m \leq 4$, then $D Q=0.25)$

$Q\left(a^{\prime}\right)=S\left(a^{\prime}\right)^{\text {or }} /$ and $R\left(a^{\prime}\right)$

[C2]: $Q\left(A_{m}\right)-Q\left(A_{1}\right)<D Q$

If $\mathrm{C} 1$ and $\mathrm{C} 2$, both accepted, Then solution is an Optimal solution, Otherwise:If [C1] is not accepted and Eq. (31) is not accepted then $A_{1}$ and $A_{m}$ are the same compromise solution. However, $\mathrm{A}_{1}$ does not have a comparative advantage, so the compromise solutions are the same. If [C2] is not accepted, the stability in decision-making is deficient, although $\mathrm{A}_{1}$ has a comparative advantage. Hence, compromise solutions of $A_{1}$ and $A_{m}$ are the same. 


\section{Appendix2.Detailed calculations for Alpha system}

Table1. Linguistic variables for comparisons

\begin{tabular}{|c|c|}
\hline Linguistic variable & Fuzzy number \\
\hline Very Low (VL) & $(1 / 2,1,3 / 2)$ \\
\hline Low (L) & $(1,3 / 2,2)$ \\
\hline Medium (M) & $(3 / 2,2,5 / 2)$ \\
\hline High (H) & $(2,5 / 2,3)$ \\
\hline Very High (VH) & $(5 / 2,3,7 / 2)$ \\
\hline Equal & $(1,1,1)$ \\
\hline
\end{tabular}

Table 2. The construct of decision model

\begin{tabular}{|c|c|c|c|c|c|}
\hline Main-criteria & \multicolumn{5}{|c|}{ Sub-criterias } \\
\hline $\begin{array}{c}\text { Agility } \\
\text { (Collective sub- } \\
\text { criterias) }\end{array}$ & $\begin{array}{c}\text { Flexibility } \\
\text { (SC1) }\end{array}$ & $\begin{array}{c}\text { Speed } \\
\text { (SC2) }\end{array}$ & $\begin{array}{c}\text { Leanness } \\
\text { (SC3) }\end{array}$ & $\begin{array}{c}\text { Learning } \\
\text { (SC4) }\end{array}$ & $\begin{array}{c}\text { Responsiveness } \\
\text { (SC5) }\end{array}$ \\
\hline $\begin{array}{c}\text { Modularity } \\
\text { (Individual sub- } \\
\text { criterias) }\end{array}$ & \multicolumn{2}{|c|}{$\begin{array}{c}\text { Expandability } \\
\text { (SC6) }\end{array}$} & \multicolumn{2}{|c|}{$\begin{array}{c}\text { Decomposability } \\
\text { (SC7) }\end{array}$} & \multicolumn{2}{c|}{$\begin{array}{c}\text { Recombinability } \\
\text { (SC8) }\end{array}$} \\
\hline
\end{tabular}

Table 3.Average of aggregated fuzzy numbers extracted from expert's opinions about collective sub-criterias

\begin{tabular}{|c|c|c|c|c|c|}
\hline & SC1 & SC2 & SC3 & SC4 & SC5 \\
\hline SC1 & $(1,1,1)$ & $(2.1,2.6,3.10)$ & $(2.3,2.8,3.3)$ & $(2.5,3.0,3.5)$ & $(1.5,2,2.5)$ \\
\hline SC2 & $(0.32,0.38,0.48)$ & $(1,1,1)$ & $(2,2.5,3.0)$ & $(2.2,2.7,3.2)$ & $(1.1,1.6,2.1)$ \\
\hline SC3 & $(0.3,0.36,0.43)$ & $(0.33,0.4,0.5)$ & $(1,1,1)$ & $(.7,1.2,1.7)$ & $(.5,1.0,1.5)$ \\
\hline SC4 & $(0.29,0.33,0.4)$ & $(0.31, .37,0.45)$ & $(0.59,0.83,1.43)$, & $(1,1,1)$ & $(.7,1.2,1.7)$ \\
\hline SC5 & $(0.4,0.5,0.67)$ & $(0.48,0.63,0.91)$ & $(0.67,1.0,2.0)$ & $(0.59,0.83,1.43)$ & $(1,1,1)$ \\
\hline
\end{tabular}

$\mathrm{S} 1=(0.24,0.36,0.54) ; \mathrm{S} 2=(0.17,0.26,0.39) ; \mathrm{S} 3=(0.07,0.13,0.21) ; \mathrm{S} 4=(0.07,0.12,0.20)$

$\mathrm{S} 5=(0.08,0.13,0.24)$

After determining of S_SCi, we need to calculate PDL of each sub criterias against each other'sas shown below.

$$
\begin{aligned}
& \mathrm{V}(\mathrm{SC} 1>=\mathrm{SCi})=1 ; \mathrm{i}=2,3,4,5 \\
& \mathrm{~V}(\mathrm{SC} 2>=\mathrm{SC} 1)=0.5999 ; \mathrm{V}(\mathrm{SC} 2>=\mathrm{SC} 3)=1 ; \mathrm{V}(\mathrm{SC} 2>=\mathrm{SC} 4)=1 ; \mathrm{V}(\mathrm{SC} 2>=\mathrm{SC} 5)=1 \\
& \mathrm{~V}(\mathrm{SC} 3>=\mathrm{SC} 1)=0 ; \mathrm{V}(\mathrm{SC} 3>=\mathrm{SC} 2)=0.21 ; \mathrm{V}(\mathrm{SC} 3>=\mathrm{SC} 4)=1 ; \mathrm{V}(\mathrm{SC} 3>=\mathrm{SC} 5)=0.99 \\
& \mathrm{~V}(\mathrm{SC} 4>=\mathrm{SC} 1)=0 ; \mathrm{V}(\mathrm{SC} 4>=\mathrm{SC} 2)=0.18 ; \mathrm{V}(\mathrm{SC} 4>=\mathrm{SC} 3)=0.94 ; \mathrm{V}(\mathrm{SC} 4>=\mathrm{SC} 5)=0.94 \\
& \mathrm{~V}(\mathrm{SC} 5>=\mathrm{SC} 1)=0.009 ; \mathrm{V}(\mathrm{SC} 5>=\mathrm{SC} 2)=0.34 ; \mathrm{V}(\mathrm{SC} 5>=\mathrm{SC} 3)=1 ; \mathrm{V}(\mathrm{SC} 5>=\mathrm{SC} 4)=1
\end{aligned}
$$

The weigh vector from refereed calculation will be: $W^{\prime}=(1,0.59,0,0,0.009)$.Therefore the normalized weight is : $\mathrm{W}=(0.62,0.37,0,0,0.01)$.These calculations also should be performed for modularity's criteria. This criteriaconsidering of experts opinionshas three sub-criteria.

Table 4.The Aggregated fuzzy numbers extracted from expert's opinions about individual sub-criterias

\begin{tabular}{|l|c|c|c|}
\hline & SC6 & SC7 & SC8 \\
\hline SC6 & $(1,1,1)$ & $(0.9,1.4,1.9)$ & $(1.2,1.7,2.2)$ \\
\hline SC7 & $(0.53,0.71,1.11)$ & $(1,1,1)$ & $(0.7,1.2,1.7)$ \\
\hline SC8 & $(0.45,0.59,0.83)$ & $(0.59,0.83,1.43)$ & $(1,1,1)$ \\
\hline
\end{tabular}


$S_{1}=(0.18,0.32,0.53) ; S_{2}=(0.30,0.45,0.71) ; S_{3}=(0.15,0.22,0.38)$

After determining of S_SCi these result can be calculated:

$\mathrm{V}\left(\mathrm{SC}_{1}>=\mathrm{SC}_{2}\right)=0.64 ; \mathrm{V}\left(\mathrm{SC}_{1}>=\mathrm{SC}_{3}\right)=1$

$\mathrm{V}\left(\mathrm{SC}_{2}>=\mathrm{SC}_{\mathrm{i}}\right)=1 ; \mathrm{i}=1,3$

$\mathrm{V}\left(\mathrm{SC}_{3}>=\mathrm{SC}_{1}\right)=0.67 ; \mathrm{V}\left(\mathrm{SC}_{3}>=\mathrm{SC}_{2}\right)=0.27$

The weigh vector from previous calculation will be: $W^{\prime}=(0.64,1,0.27)$ Therefore the normalized weight is: $\mathrm{W}=(0.34,0.52,0.14)$. The final weight according to eq

Table 5.The normalized weights of main-criteria and sub-criterias according to fuzzy AHP techniques

\begin{tabular}{|c|c|c|c|c|c|}
\hline \multirow{2}{*}{$\begin{array}{c}\text { Agility's sub- } \\
\text { criterias }\end{array}$} & SC1 & SC2 & SC3 & SC4 & SC5 \\
\cline { 2 - 5 } & Flexibility & Speed & Leanness & Learning & Responsive \\
\hline \multirow{3}{*}{$\begin{array}{c}\text { Modularity's } \\
\text { sub-criterias }\end{array}$} & 0.62 & 0.37 & 0 & 0 & 0.01 \\
\cline { 2 - 4 } & SC6 & SC7 & SC8 & \\
\cline { 2 - 4 } & 0.34 & Decomposability & Recombinability & \\
\hline
\end{tabular}

Table 6. Average of aggregated fuzzy numbers about pairwise comparisons of alternatives in flexibility subcriteria

\begin{tabular}{|c|c|c|c|c|c|}
\hline & A1 & A2 & A3 & A4 & A5 \\
\hline A1 & $(1,1,1)$ & $(2.1,2.6,3.1)$ & $(1.9,2.4,2.9)$ & $(2.2,2.7,3.2)$ & $(1.5,2,2.5)$ \\
\hline A2 & $(0.32,0.38,0.48)$ & $(1,1,1)$ & $(2.1,2.6,3.1)$ & $(2.4,2.9,3.4)$ & $(2.1,2.6,3.1)$ \\
\hline A3 & $(0.34,0.42,0.53)$ & $(0.32,0.38,0.48)$ & $(1,1,1)$ & $(1.1,1.6,2.1)$ & $(1.3,1.8,2.3)$ \\
\hline A4 & $(0.31,0.37,0.45)$ & $(0.29,0.34,0.42)$ & $(0.48,0.63,0.91)$ & $(1,1,1)$ & $(2.5,3,3.5)$ \\
\hline A5 & $(0.40,0.50,0.67)$ & $(0.32,0.38,0.48)$ & $(0.43,0.56,0.77)$ & $(0.29,0.33,0.40)$ & $(1,1,1)$ \\
\hline
\end{tabular}

Table 7. Average of aggregated fuzzy numbers about pairwise comparisons of alternatives in speed subcriteria

\begin{tabular}{|c|c|c|c|c|c|}
\hline & A1 & A2 & A3 & A4 & A5 \\
\hline A1 & $(1,1,1)$ & $(2,2.5,3)$ & $(1.9,2.4,2.9)$ & $(2,2.5,3)$ & $(1.5,2.0,2.5)$ \\
\hline A2 & $(0.33,0.4,0.5)$ & $(1,1,1)$ & $(2.3,2.8,3.3)$ & $(2.4,2.9,3.4)$ & $(2.1,2.6,3.1)$ \\
\hline A3 & $(0.34,0.42,0.53)$ & $(0.3,0.36,0.43)$ & $(1,1,1)$ & $(1.6,2.1,2.6)$ & $(1.2,1.7,2.2)$ \\
\hline A4 & $(0.33,0.4,0.5)$ & $(0.29,0.34,0.42)$ & $(0.38,0.48,0.63)$ & $(1,1,1)$ & $(2.5,3,3.5)$ \\
\hline A5 & $(0.4,0.5,0.67)$ & $(0.32,0.38,0.48)$ & $(0.45,0.59,0.83)$ & $(0.29,0.33,0.4)$ & $(1,1,1)$ \\
\hline
\end{tabular}

Table 8. Average of aggregated fuzzy numbers about pairwise comparisons of alternatives in Leanness subcriteria

\begin{tabular}{|c|c|c|c|c|c|}
\hline & A1 & A2 & A3 & A4 & A5 \\
\hline A1 & $(1,1,1)$ & $(2.1,2.6,3.1)$ & $(1.8,2.3,2.8)$ & $(2.1,2.6,3.1)$ & $(1.5,2.0,2.5)$ \\
\hline A2 & $(0.32,0.38,0.48)$ & $(1,1,1)$ & $(2.1,2.6,3.1)$ & $(2.4,2.9,3.4)$ & $(2.2,2.7,3.2)$ \\
\hline A3 & $(0.36,0.43,0.56)$ & $(0.32,0.38,0.48)$ & $(1,1,1)$ & $(1.7,2.2,2.7)$ & $(1.6,2.1,2.6)$ \\
\hline A4 & $(0.32,0.38,0.48)$ & $(0.29,0.34,0.42)$ & $(0.37,0.45,0.59)$ & $(1,1,1)$ & $(2.5,3.0,3.5)$ \\
\hline A5 & $(0.4,0.5,0.67)$ & $(0.31,0.37,0.45)$ & $(0.38,0.48,0.63)$ & $(0.29,0.33,0.4)$ & $(1,1,1)$ \\
\hline
\end{tabular}

Table 9.Average of aggregated fuzzy numbers about pairwise comparisons of alternatives in Learning subcriteria

\begin{tabular}{|l|l|l|l|l|l|}
\hline & A1 & A2 & A3 & A4 & A5 \\
\hline
\end{tabular}




\begin{tabular}{|c|c|c|c|c|c|}
\hline A1 & $(1,1,1)$ & $(2.2,2.7,3.2)$ & $(2.1,2.6,3.1)$ & $(2.4,2.9,3.4)$ & $(1.9,2.4,2.9)$ \\
\hline A2 & $(0.31,0.37,0.45)$ & $(1,1,1)$ & $(2.1,2.6,3.1)$ & $(2.4,2.9,3.4)$ & $(2.1,2.6,3.1)$ \\
\hline A3 & $(0.32,0.38,0.48)$ & $(0.32,0.38,0.48)$ & $(1,1,1)$ & $(1.3,1.8,2.3)$ & $(1.4,1.9,2.4)$ \\
\hline A4 & $(0.29,0.34,0.42)$ & $(0.29,0.34,0.42)$ & $(0.43,0.56,0.77)$ & $(1,1,1)$ & $(1.9,2.4,2.9)$ \\
\hline A5 & $(0.34,0.42,0.53)$ & $(0.32,0.38,0.48)$ & $(0.42,0.53,0.71)$ & $(0.34,0.42,0.53)$ & $(1,1,1)$ \\
\hline
\end{tabular}

Table 10. Average of aggregated fuzzy numbers about pairwise comparisons of alternatives in Responsivenesssub-criteria

\begin{tabular}{|c|c|c|c|c|c|}
\hline & A1 & A2 & A3 & A4 & A5 \\
\hline A1 & $(1,1,1)$ & $(1.8,2.3,2.8)$ & $(1.6,2.1,2.6)$ & $(1.2,1.7,2.2)$ & $(1.4,1.9,2.4)$ \\
\hline A2 & $(0.36,0.43,0.56)$ & $(1,1,1)$ & $(2.4,2.9,3.4)$ & $(2.4,2.9,3.4)$ & $(2.3,2.8,3.3)$ \\
\hline A3 & $(0.38,0.48,0.63)$ & $(0.29,0.34,0.42)$ & $(1,1,1)$ & $(1.1,1.6,2.1)$ & $(1.3,1.8,2.3)$ \\
\hline A4 & $(0.45,0.59,0.83)$ & $(0.29,0.34,0.42)$ & $(0.48,0.63,0.91)$ & $(1,1,1)$ & $(2.5,3.0,3.5)$ \\
\hline A5 & $(0.42,0.53,0.71)$ & $(0.3,0.36,0.43)$ & $(0.43,0.56,0.77)$ & $(0.29,0.34,0.40)$ & $(1,1,1)$ \\
\hline
\end{tabular}

Table 11. Average of aggregated fuzzy numbers about pairwise comparisons of alternatives in Expanability subcriteria

\begin{tabular}{|c|c|c|c|c|c|}
\hline & A1 & A2 & A3 & A4 & A5 \\
\hline A1 & $(1,1,1)$ & $(1.8,2.3,2.8)$ & $(1.4,1.9,2.4)$ & $(1.7,2.2,2.7)$ & $(0.8,1.3,1.8)$ \\
\hline A2 & $(0.36,0.43,0.56)$ & $(1,1,1)$ & $(2.3,2.8,3.3)$ & $(2.4,2.9,3.4)$ & $(2.1,2.6,3.1)$ \\
\hline A3 & $(0.42,0.53,0.71)$ & $(0.3,0.36,0.43)$ & $(1,1,1)$ & $(1.1,1.6,2.1)$ & $(1.3,1.8,2.3)$ \\
\hline A4 & $(0.37,0.45,0.59)$ & $(0.29,0.34,0.42)$ & $(0.48,0.63,0.91)$ & $(1,1,1)$ & $(2.5,3.0,3.5)$ \\
\hline A5 & $(0.56,0.77,1.25)$ & $(0.32,0.38,0.48)$ & $(0.43,0.56,0.77)$ & $(0.29,0.34,0.40)$ & $(1,1,1)$ \\
\hline
\end{tabular}

Table 12.Average of aggregated fuzzy numbers about pairwise comparisons of alternatives in Decomposability sub-criteria

\begin{tabular}{|c|c|c|c|c|c|}
\hline & A1 & A2 & A3 & A4 & A5 \\
\hline A1 & $(1,1,1)$ & $(0.7,1.2,1.7)$ & $(1,1.5,2)$ & $(1,1.5,2)$ & $(0.7,1.2,1.7)$ \\
\hline A2 & $(0.59,0.83,1.43)$ & $(1,1,1)$ & $(2.4,2.9,3.4)$ & $(2.4,2.9,3.4)$ & $(2.0,2.5,3)$ \\
\hline A3 & $(0.5,0.67,1)$ & $(0.29,0.34,0.42)$ & $(1,1,1)$ & $(1.1,1.6,2.1)$ & $(1,1.5,2)$ \\
\hline A4 & $(0.5,0.67,1)$ & $(0.29,0.34,0.42)$ & $(0.48,0.63,0.91)$ & $(1,1,1)$ & $(2.5,3.0,3.5)$ \\
\hline A5 & $(0.59,0.83,1.43)$ & $(0.33,0.4,0.5)$ & $(0.5,0.67,1)$ & $(0.29,0.34,0.40)$ & $(1,1,1)$ \\
\hline
\end{tabular}

Table 13.Average of aggregated fuzzy numbers about pairwise comparisons of alternatives in Recombinability sub-criteria

\begin{tabular}{|c|c|c|c|c|c|}
\hline & A1 & A2 & A3 & A4 & A5 \\
\hline A1 & $(1,1,1)$ & $(2.1,2.6,3.1)$ & $(1.7,2.2,2.7)$ & $(2,2.5,3)$ & $(0.9,1.4,1.9)$ \\
\hline A2 & $(0.32,0.38,0.48)$ & $(1,1,1)$ & $(1,1.5,2)$ & $(2.4,2.9,3.4)$ & $(2.1,2.6,3.1)$ \\
\hline A3 & $(0.37,0.45,0.59)$ & $(0.31,0.37,0.45)$ & $(1,1,1)$ & $(1,1.5,2)$ & $(1.2,1.7,2.2)$ \\
\hline A4 & $(0.33,0.4,0.5)$ & $(0.29,0.34,0.42)$ & $(0.5,0.67,1)$ & $(1,1,1)$ & $(2.5,3.0,3.5)$ \\
\hline A5 & $(0.53,0.71,1.11)$ & $(0.32,0.38,0.48)$ & $(0.45,0.59,0.83)$ & $(0.29,0.33,0.40)$ & $(1,1,1)$ \\
\hline
\end{tabular}

For table 6. Results will be:

$\mathrm{S} 1=(8.7,10.7,12.7)^{*}(27.72,33.50,39.77)^{-1}=(0.22,0.32,0.46)$

$\mathrm{S} 2=(7.92,9.48,11.08)^{*}(27.72,33.50,39.77)^{-1}=(0.2,0.28,0.4)$

$\mathrm{S} 3=(4.07,5.20,6.40)^{*}(27.72,33.50,39.77)^{-1}=(0.10,0.16,0.23)$

$\mathrm{S} 4=(4.58,5.34,6.28)^{*}(27.72,33.50,39.77)^{-1}=(0.12,0.16,0.23)$

$\mathrm{S} 5=(2.44,2.77,3.31)^{*}(27.72,33.50,39.77)^{-1}=(0.06,0.08,0.12)$

Table 14.Calculation of possibility's degree of relative largeness of Si for table 6

\begin{tabular}{|c|c|c|c|c|c|}
\hline \multicolumn{2}{|c|}{$\mathrm{V}\left[\mathrm{S}_{1}>=\mathrm{S}_{i}\right]$} & \multicolumn{2}{c|}{$\mathrm{V}\left[\mathrm{S}_{2}>=\mathrm{S}_{\mathrm{i}}\right]$} & \multicolumn{2}{c|}{$\mathrm{V}\left[\mathrm{S}_{3}>=\mathrm{S}_{\mathrm{i}}\right]$} \\
\hline $\mathrm{V}\left[\mathrm{S}_{1}>=\mathrm{S}_{2}\right]$ & 1 & $\mathrm{~V}\left[\mathrm{~S}_{2}>=\mathrm{S}_{1}\right]$ & 0.83 & $\mathrm{~V}\left[\mathrm{~S}_{3}>=\mathrm{S}_{1}\right]$ & 0.07 \\
\hline $\mathrm{V}\left[\mathrm{S}_{1}>=\mathrm{S}_{3}\right]$ & 1 & $\mathrm{~V}\left[\mathrm{~S}_{2}>=\mathrm{S}_{3}\right]$ & 1 & $\mathrm{~V}\left[\mathrm{~S}_{3}>=\mathrm{S}_{2}\right]$ & 0.2 \\
\hline $\mathrm{V}\left[\mathrm{S}_{1}>=\mathrm{S}_{4}\right]$ & 1 & $\mathrm{~V}\left[\mathrm{~S}_{2}>=\mathrm{S}_{4}\right]$ & 1 & $\mathrm{~V}\left[\mathrm{~S}_{3}>=\mathrm{S}_{4}\right]$ & 0.96 \\
\hline
\end{tabular}


Peyman mohammady, Amin Amid / TJMCS Vol .1 No.4 (2010) 413-434

\begin{tabular}{|c|c|c|c|c|c|}
\hline $\mathrm{V}\left[\mathrm{S}_{1}>=\mathrm{S}_{5}\right]$ & 1 & $\mathrm{~V}\left[\mathrm{~S}_{2}>=\mathrm{S}_{5}\right]$ & 1 & $\mathrm{~V}\left[\mathrm{~S}_{3}>=\mathrm{S}_{5}\right]$ & 1 \\
\cline { 1 - 4 } $\mathrm{V}\left[\mathrm{S}_{4}>=\mathrm{S}_{\mathrm{i}}\right]$ & \multicolumn{2}{|c|}{$\mathrm{V}\left[\mathrm{S}_{5}>=\mathrm{S}_{\mathrm{i}}\right]$} & & \\
\cline { 1 - 4 } $\mathrm{V}[\mathrm{S} 4>=\mathrm{S} 1]$ & 0.046 & $\mathrm{~V}[\mathrm{~S} 5>=\mathrm{S} 1]$ & 0 & & \\
\cline { 1 - 4 } $\mathrm{V}[\mathrm{S} 4>=\mathrm{S} 2]$ & 0.18 & $\mathrm{~V}[\mathrm{~S} 5>=\mathrm{S} 2]$ & 0 & & \\
\cline { 1 - 4 } $\mathrm{V}[\mathrm{S} 4>=\mathrm{S} 3]$ & 1 & $\mathrm{~V}[\mathrm{~S} 5>=\mathrm{S} 3]$ & 0.19 & & \\
\cline { 1 - 4 } $\mathrm{V}[\mathrm{S} 4>=\mathrm{S} 5]$ & 1 & $\mathrm{~V}[\mathrm{~S} 5>=\mathrm{S} 4]$ & 0.05 & & \\
\end{tabular}

Table 15.calculation of possibility's degree of largeness of $\mathrm{S}_{\mathrm{i}}$ mainly, for table 6

\begin{tabular}{|c|c|}
\hline \multicolumn{2}{|c|}{$\mathrm{V}\left[\mathrm{S}>=\mathrm{S}_{\mathrm{i}}\right]$} \\
\hline $\mathrm{V}\left[\mathrm{S}_{1}>=\mathrm{S}_{\mathrm{i}}\right]$ & 1 \\
\hline $\mathrm{V}\left[\mathrm{S}_{2}>=\mathrm{S}_{\mathrm{i}}\right]$ & 0.83 \\
\hline $\mathrm{V}\left[\mathrm{S}_{3}>=\mathrm{S}_{\mathrm{i}}\right]$ & 0.07 \\
\hline $\mathrm{V}\left[\mathrm{S}_{4}>=\mathrm{S}_{\mathrm{i}}\right]$ & 0.046 \\
\hline $\mathrm{V}\left[\mathrm{S}_{5}>=\mathrm{S}_{\mathrm{i}}\right]$ & 0 \\
\hline
\end{tabular}

Table 16.Average of aggregated opinions extract from $S_{i}$ based on Eq. (11)

\begin{tabular}{|c|c|c|c|c|c|c|}
\hline \multicolumn{7}{|c|}{ Agility - Collective main-criteria } \\
\hline & & A1 & A2 & A3 & A4 & A5 \\
\hline \multirow{5}{*}{$\widetilde{C C}=$} & SC1 & $(0.22,0.32,0.46)$ & $(0.2,0.28,0.4)$ & $(0.10,0.16,0.23)$ & $(0.12,0.16,0.23)$ & $(0.06,0.08,0.12)$ \\
\hline & SC2 & $(0.21,0.31,0.44)$ & $(0.2,0.29,0.4)$ & $(0.11,0.17,0.24)$ & $(0.11,0.15,0.22)$ & $(0.06,0.08,0.12)$ \\
\hline & SC3 & $(0.21,0.31,0.44)$ & $(0.2,0.28,0.39)$ & $(0.12,0.18,0.26)$ & $(0.11,0.15,0.21)$ & $(0.06,0.08,0.11)$ \\
\hline & SC4 & $(0.24,0.34,0.48)$ & $(0.2,0.28,0.39)$ & $(0.11,0.16,0.24)$ & $(0.1,0.14,0.2)$ & $(0.06,0.08,0.11)$ \\
\hline & SC5 & $(0.18,0.28,0.41)$ & $(0.22,0.31,0.44)$ & $(0.1,0.16,0.24)$ & $(0.12,0.17,0.25)$ & $(0.06,0.09,0.12)$ \\
\hline \multicolumn{7}{|c|}{ Modularity - Individual main-criteria } \\
\hline \multirow{3}{*}{$\mp C$} & SC6 & $(0.17,0.27,0.41)$ & $(0.21,0.3,0.43)$ & $(0.11,0.16,0.25)$ & $(0.12,0.17,0.24)$ & $(0.07,0.09,0.15)$ \\
\hline & SC7 & $(0.11,0.21,0.35)$ & $(0.22,0.33,0.51)$ & $(0.1,0.17,0.27)$ & $(0.12,0.18,0.28)$ & $(0.07,0.11,0.18)$ \\
\hline & SC8 & $(0.2,0.3,0.44)$ & $(0.2,0.29,0.42)$ & $(0.1,0.15,0.23)$ & $(0.12,0.17,0.24)$ & $(0.07,0.09,0.14)$ \\
\hline
\end{tabular}

Table 17.fuzzy best value (FBV) and fuzzy worst value (FWV) per each criteria

\begin{tabular}{|c|c|c|c|c|c|c|}
\hline & \multicolumn{3}{|c|}{${ }^{*} f$} & \multicolumn{3}{|c|}{ if } \\
\hline SC1 & 0.22 & 0.32 & 0.46 & 0.06 & 0.08 & 0.12 \\
\hline SC2 & 0.21 & 0.31 & 0.44 & 0.06 & 0.08 & 0.12 \\
\hline SC3 & 0.21 & 0.31 & 0.44 & 0.06 & 0.08 & 0.11 \\
\hline SC4 & 0.24 & 0.34 & 0.48 & 0.06 & 0.08 & 0.11 \\
\hline SC5 & 0.22 & 0.31 & 0.44 & 0.06 & 0.09 & 0.12 \\
\hline & \multicolumn{3}{|c|}{${ }^{*} f$} & \multicolumn{3}{|c|}{ if } \\
\hline SC6 & 0.21 & 0.30 & 0.43 & 0.07 & 0.09 & 0.15 \\
\hline SC7 & 0.22 & 0.33 & 0.51 & 0.07 & 0.11 & 0.18 \\
\hline SC8 & 0.20 & 0.30 & 0.44 & 0.07 & 0.09 & 0.14 \\
\hline
\end{tabular}

Table 18. Calculation of $S_{j}$ and $R_{j}$ for whole of sub-criterias

\begin{tabular}{|c|c|c|c|c|c|c|c|c|c|c|c|c|c|c|c|c|}
\hline \multicolumn{17}{|c|}{ Agility - Collective main-criteria } \\
\hline & & \multicolumn{3}{|c|}{ A1 } & \multicolumn{3}{|c|}{ A2 } & \multicolumn{3}{|c|}{ A3 } & \multicolumn{3}{|c|}{ A4 } & \multicolumn{3}{|c|}{ A5 } \\
\hline \multirow{5}{*}{$\approx C$} & SC1 & 0.000 & $\begin{array}{c}0.00 \\
0\end{array}$ & $\begin{array}{c}0.00 \\
0\end{array}$ & $\begin{array}{c}0.07 \\
7\end{array}$ & $\begin{array}{c}0.09 \\
5\end{array}$ & $\begin{array}{c}0.10 \\
7\end{array}$ & $\begin{array}{c}0.45 \\
9\end{array}$ & $\begin{array}{c}0.43 \\
0\end{array}$ & $\begin{array}{c}0.41 \\
6\end{array}$ & $\begin{array}{c}0.40 \\
8\end{array}$ & $\begin{array}{c}0.41 \\
9\end{array}$ & $\begin{array}{c}0.42 \\
4\end{array}$ & $\begin{array}{c}0.62 \\
0\end{array}$ & $\begin{array}{c}0.62 \\
0\end{array}$ & $\begin{array}{c}0.62 \\
0\end{array}$ \\
\hline & SC2 & 0.000 & $\begin{array}{c}0.00 \\
0\end{array}$ & $\begin{array}{c}0.00 \\
0\end{array}$ & $\begin{array}{c}0.01 \\
7\end{array}$ & $\begin{array}{c}0.03 \\
4\end{array}$ & $\begin{array}{c}0.04 \\
5\end{array}$ & $\begin{array}{c}0.24 \\
6\end{array}$ & $\begin{array}{c}0.23 \\
5\end{array}$ & $\begin{array}{c}0.23 \\
1\end{array}$ & $\begin{array}{c}0.24 \\
2\end{array}$ & $\begin{array}{c}0.25 \\
2\end{array}$ & $\begin{array}{c}0.26 \\
1\end{array}$ & $\begin{array}{c}0.37 \\
0\end{array}$ & $\begin{array}{c}0.37 \\
0\end{array}$ & $\begin{array}{c}0.37 \\
0\end{array}$ \\
\hline & SC3 & 0.000 & $\begin{array}{c}0.00 \\
0 \\
\end{array}$ & $\begin{array}{c}0.00 \\
0\end{array}$ & $\begin{array}{c}0.00 \\
0\end{array}$ & $\begin{array}{c}0.00 \\
0\end{array}$ & $\begin{array}{c}0.00 \\
0\end{array}$ & $\begin{array}{c}0.00 \\
0\end{array}$ & $\begin{array}{c}0.00 \\
0\end{array}$ & $\begin{array}{c}0.00 \\
0\end{array}$ & $\begin{array}{c}0.00 \\
0\end{array}$ & $\begin{array}{c}0.00 \\
0\end{array}$ & $\begin{array}{c}0.00 \\
0\end{array}$ & $\begin{array}{c}0.00 \\
0\end{array}$ & $\begin{array}{c}0.00 \\
0\end{array}$ & $\begin{array}{c}0.00 \\
0\end{array}$ \\
\hline & SC4 & 0.000 & $\begin{array}{c}0.00 \\
0\end{array}$ & $\begin{array}{c}0.00 \\
0\end{array}$ & $\begin{array}{c}0.00 \\
0\end{array}$ & $\begin{array}{c}0.00 \\
0\end{array}$ & $\begin{array}{c}0.00 \\
0\end{array}$ & $\begin{array}{c}0.00 \\
0\end{array}$ & $\begin{array}{c}0.00 \\
0\end{array}$ & $\begin{array}{c}0.00 \\
0\end{array}$ & $\begin{array}{c}0.00 \\
0\end{array}$ & $\begin{array}{c}0.00 \\
0\end{array}$ & $\begin{array}{c}0.00 \\
0\end{array}$ & $\begin{array}{c}0.00 \\
0\end{array}$ & $\begin{array}{c}0.00 \\
0\end{array}$ & $\begin{array}{c}0.00 \\
0\end{array}$ \\
\hline & SC5 & 0.002 & $\begin{array}{c}0.00 \\
1\end{array}$ & $\begin{array}{c}0.00 \\
1\end{array}$ & $\begin{array}{c}0.00 \\
0\end{array}$ & $\begin{array}{c}0.00 \\
0\end{array}$ & $\begin{array}{c}0.00 \\
0\end{array}$ & $\begin{array}{c}0.00 \\
7\end{array}$ & $\begin{array}{c}0.00 \\
7\end{array}$ & $\begin{array}{c}0.00 \\
6\end{array}$ & $\begin{array}{c}0.00 \\
6\end{array}$ & $\begin{array}{c}0.00 \\
6\end{array}$ & $\begin{array}{c}0.00 \\
6\end{array}$ & $\begin{array}{c}0.01 \\
0\end{array}$ & $\begin{array}{c}0.01 \\
0\end{array}$ & $\begin{array}{c}0.01 \\
0\end{array}$ \\
\hline \multicolumn{2}{|c|}{$\tilde{\boldsymbol{S}}$} & 0.00 & 0.00 & 0.00 & 0.00 & 0.09 & 0.13 & 0.15 & 0.71 & 0.67 & 0.65 & 0.66 & 0.68 & 0.69 & 1.00 & 1.00 \\
\hline
\end{tabular}


Peyman mohammady, Amin Amid / TJMCS Vol .1 No.4 (2010) 413-434

\begin{tabular}{|c|c|c|c|c|c|c|c|c|c|c|c|c|c|c|c|c|}
\hline \multicolumn{2}{|c|}{$\tilde{R} \boldsymbol{R}$} & 0.002 & $\begin{array}{c}0.00 \\
2\end{array}$ & $\begin{array}{c}0.00 \\
1\end{array}$ & $\begin{array}{c}0.00 \\
1\end{array}$ & $\begin{array}{c}0.07 \\
7\end{array}$ & $\begin{array}{c}0.09 \\
5\end{array}$ & $\begin{array}{c}0.10 \\
7\end{array}$ & $\begin{array}{c}0.45 \\
9\end{array}$ & $\begin{array}{c}0.43 \\
0\end{array}$ & $\begin{array}{c}0.41 \\
6\end{array}$ & $\begin{array}{c}0.40 \\
8\end{array}$ & $\begin{array}{c}0.41 \\
9\end{array}$ & $\begin{array}{c}0.42 \\
4\end{array}$ & $\begin{array}{c}0.62 \\
0\end{array}$ & $\begin{array}{c}0.62 \\
0\end{array}$ \\
\hline \multicolumn{17}{|c|}{ Modularity - Individual main-criteria } \\
\hline \multirow{3}{*}{$\mp C$} & SC6 & 0.089 & $\begin{array}{c}0.05 \\
3\end{array}$ & $\begin{array}{c}0.03 \\
0\end{array}$ & $\begin{array}{c}0.00 \\
0\end{array}$ & $\begin{array}{c}0.00 \\
0\end{array}$ & $\begin{array}{c}0.00 \\
0\end{array}$ & $\begin{array}{c}0.24 \\
7\end{array}$ & $\begin{array}{c}0.22 \\
6\end{array}$ & $\begin{array}{c}0.21 \\
9\end{array}$ & $\begin{array}{c}0.21 \\
5\end{array}$ & $\begin{array}{c}0.21 \\
9\end{array}$ & $\begin{array}{c}0.22 \\
5\end{array}$ & $\begin{array}{c}0.34 \\
0\end{array}$ & $\begin{array}{c}0.34 \\
0\end{array}$ & $\begin{array}{c}0.34 \\
0\end{array}$ \\
\hline & SC7 & 0.365 & $\begin{array}{c}0.28 \\
1\end{array}$ & $\begin{array}{c}0.25 \\
2\end{array}$ & $\begin{array}{c}0.00 \\
0\end{array}$ & $\begin{array}{c}0.00 \\
0\end{array}$ & $\begin{array}{c}0.00 \\
0\end{array}$ & $\begin{array}{c}0.41 \\
1\end{array}$ & $\begin{array}{c}0.37 \\
8\end{array}$ & $\begin{array}{c}0.37 \\
6\end{array}$ & $\begin{array}{c}0.33 \\
1\end{array}$ & $\begin{array}{c}0.33 \\
9\end{array}$ & $\begin{array}{c}0.35 \\
6\end{array}$ & $\begin{array}{c}0.52 \\
0\end{array}$ & $\begin{array}{c}0.52 \\
0\end{array}$ & $\begin{array}{c}0.52 \\
0\end{array}$ \\
\hline & SC8 & 0.008 & $\begin{array}{c}0.00 \\
0\end{array}$ & $\begin{array}{c}0.00 \\
0\end{array}$ & $\begin{array}{c}0.00 \\
0\end{array}$ & $\begin{array}{c}0.00 \\
2\end{array}$ & $\begin{array}{c}0.00 \\
9\end{array}$ & $\begin{array}{c}0.10 \\
7\end{array}$ & $\begin{array}{c}0.09 \\
8\end{array}$ & $\begin{array}{c}0.09 \\
7\end{array}$ & $\begin{array}{c}0.08 \\
7\end{array}$ & $\begin{array}{c}0.09 \\
0\end{array}$ & $\begin{array}{c}0.09 \\
4\end{array}$ & $\begin{array}{c}0.14 \\
0\end{array}$ & $\begin{array}{c}0.14 \\
0\end{array}$ & $\begin{array}{c}0.14 \\
0\end{array}$ \\
\hline \multicolumn{2}{|c|}{$\tilde{\not} \boldsymbol{S}$} & 0.463 & $\begin{array}{c}0.46 \\
3\end{array}$ & $\begin{array}{c}0.33 \\
4\end{array}$ & $\begin{array}{c}0.28 \\
2 \\
\end{array}$ & $\begin{array}{c}0.00 \\
0\end{array}$ & $\begin{array}{c}0.00 \\
2\end{array}$ & $\begin{array}{c}0.00 \\
9\end{array}$ & $\begin{array}{c}0.76 \\
5\end{array}$ & $\begin{array}{c}0.70 \\
3 \\
\end{array}$ & $\begin{array}{c}0.69 \\
2 \\
\end{array}$ & $\begin{array}{c}0.63 \\
4\end{array}$ & $\begin{array}{c}0.64 \\
8 \\
\end{array}$ & $\begin{array}{c}0.67 \\
5\end{array}$ & $\begin{array}{c}1.00 \\
0\end{array}$ & $\begin{array}{c}1.00 \\
0\end{array}$ \\
\hline \multicolumn{2}{|c|}{ 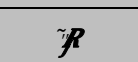 } & 0.365 & $\begin{array}{c}0.36 \\
5\end{array}$ & $\begin{array}{c}0.28 \\
1\end{array}$ & $\begin{array}{c}0.25 \\
2\end{array}$ & $\begin{array}{c}0.00 \\
0\end{array}$ & $\begin{array}{c}0.00 \\
2\end{array}$ & $\begin{array}{c}0.00 \\
9\end{array}$ & $\begin{array}{c}0.41 \\
1\end{array}$ & $\begin{array}{c}0.37 \\
8\end{array}$ & $\begin{array}{c}0.37 \\
6\end{array}$ & $\begin{array}{c}0.33 \\
1\end{array}$ & $\begin{array}{c}0.33 \\
9\end{array}$ & $\begin{array}{c}0.35 \\
6\end{array}$ & $\begin{array}{c}0.52 \\
0\end{array}$ & $\begin{array}{c}0.52 \\
0\end{array}$ \\
\hline
\end{tabular}

Table 19. Calculation of $S_{j}^{*}, S_{j}, R_{j}^{*}$ and $R_{j}$ for each of main-criterias

\begin{tabular}{|c|c|c|c|c|c|}
\hline \multicolumn{6}{|c|}{$\begin{array}{l}\text { The first section of decision-making } \\
\text { (Agility: only collective main-criteria) }\end{array}$} \\
\hline 0.00 & 0.00 & 0.00 & 1.00 & 1.00 & 1.00 \\
\hline 0.00 & 0.00 & 0.00 & 0.62 & 0.62 & 0.62 \\
\hline \multicolumn{6}{|c|}{$\begin{array}{l}\text { The second section of decision-making } \\
\text { (Modularity: only Individual main-criteria) }\end{array}$} \\
\hline 0.00 & 0.00 & 0.01 & 1.00 & 1.00 & 1.00 \\
\hline 0.00 & 0.00 & 0.01 & 0.52 & 0.52 & 0.52 \\
\hline
\end{tabular}

Table 20. Final calculation and rancked list of alternatives

\begin{tabular}{|c|c|c|c|c|c|c|c|c|c|c|c|c|c|c|c|}
\hline & \multicolumn{3}{|c|}{$\mathbf{A}_{1}$} & \multicolumn{3}{|c|}{$\mathbf{A}_{2}$} & \multicolumn{3}{|c|}{$\mathbf{A}_{3}$} & \multicolumn{3}{|c|}{$\mathbf{A}_{4}$} & \multicolumn{3}{|c|}{$\mathbf{A}_{5}$} \\
\hline R & $\begin{array}{c}0.00 \\
0\end{array}$ & $\begin{array}{c}0.00 \\
0\end{array}$ & $\begin{array}{c}0.00 \\
0 \\
\end{array}$ & $\begin{array}{c}0.09 \\
3 \\
\end{array}$ & $\begin{array}{c}0.12 \\
9 \\
\end{array}$ & $\begin{array}{c}0.15 \\
3\end{array}$ & $\begin{array}{c}0.71 \\
3 \\
\end{array}$ & $\begin{array}{c}0.67 \\
2 \\
\end{array}$ & $\begin{array}{c}0.65 \\
4 \\
\end{array}$ & $\begin{array}{c}0.65 \\
6 \\
\end{array}$ & $\begin{array}{c}0.67 \\
7 \\
\end{array}$ & $\begin{array}{c}0.69 \\
0 \\
\end{array}$ & $\begin{array}{c}1.00 \\
0 \\
\end{array}$ & $\begin{array}{c}1.00 \\
0 \\
\end{array}$ & $\begin{array}{c}1.00 \\
0\end{array}$ \\
\hline$Q_{i^{\prime}}$ & \multicolumn{3}{|c|}{0.000} & \multicolumn{3}{|c|}{0.125} & \multicolumn{3}{|c|}{0.680} & \multicolumn{3}{|c|}{0.674} & \multicolumn{3}{|c|}{1.000} \\
\hline$\tilde{R}$ & $\begin{array}{c}0.67 \\
8\end{array}$ & $\begin{array}{c}0.51 \\
8\end{array}$ & $\begin{array}{c}0.45 \\
5\end{array}$ & $\begin{array}{c}0.00 \\
0\end{array}$ & $\begin{array}{c}0.00 \\
0\end{array}$ & $\begin{array}{c}0.00 \\
0\end{array}$ & $\begin{array}{c}0.78 \\
8\end{array}$ & $\begin{array}{c}0.72 \\
4\end{array}$ & $\begin{array}{c}0.71 \\
5\end{array}$ & $\begin{array}{c}0.63 \\
7\end{array}$ & $\begin{array}{c}0.65 \\
0\end{array}$ & $\begin{array}{c}0.67 \\
7\end{array}$ & $\begin{array}{c}1.00 \\
0\end{array}$ & $\begin{array}{c}1.00 \\
0\end{array}$ & $\begin{array}{c}1.00 \\
0\end{array}$ \\
\hline$Q_{i^{\prime \prime}}$ & \multicolumn{3}{|c|}{0.551} & \multicolumn{3}{|c|}{0.000} & \multicolumn{3}{|c|}{0.743} & \multicolumn{3}{|c|}{0.655} & \multicolumn{3}{|c|}{1.000} \\
\hline$\tilde{R}$ & \multicolumn{3}{|c|}{0.330} & \multicolumn{3}{|c|}{0.050} & \multicolumn{3}{|c|}{0.717} & \multicolumn{3}{|c|}{0.662} & \multicolumn{3}{|c|}{1.000} \\
\hline Rank* & \multicolumn{3}{|c|}{2} & \multicolumn{3}{|c|}{ 1* } & \multicolumn{3}{|c|}{4} & \multicolumn{3}{|c|}{3} & \multicolumn{3}{|c|}{5} \\
\hline
\end{tabular}

